\title{
LC-HRMS Based Non-Targeted Metabolomic Profiling of Wheat (Triticum aestivum L.) under Post-Anthesis Drought Stress
}

\author{
Mohammad Atikur Rahman'1*, Masum Akond1*, Md Ali Babar1, Chris Beecher ${ }^{2,3}$, John Erickson"1, \\ Kayla Thomason', Felice A De Jong ${ }^{3}$, Richard Esten Mason ${ }^{4}$
}

\author{
${ }^{1}$ Agronomy Department, IFAS, University of Florida, Gainesville, FL, USA \\ ${ }^{2}$ Southeast Center for Integrated Metabolomics (SECIM), University of Florida, Gainesville, FL, USA \\ ${ }^{3}$ IROA Technologies LLC, Ann Arbor, MI, USA \\ ${ }^{4}$ Crop, Soil and Environmental Sciences, University of Arkansas, Fayetteville, AR, USA \\ Email:mababar@ufl.edu
}

How to cite this paper: Rahman, M.A. Akond, M., Babar, M.A., Beecher, C., Erickson, J., Thomson, K., De Jong, F.A, and Mason, R.E. (2017) LC-HRMS Based Non-Targeted Metabolomic Profiling of Wheat (Triticum aestivum L.) under Post-Anthesis Drought Stress. American Journal of Plant Sciences, 8, 3024-3061. https://doi.org/10.4236/ajps.2017.812205

Received: September 25, 2017

Accepted: November 12, 2017

Published: November 15, 2017

Copyright (c) 2017 by authors and Scientific Research Publishing Inc. This work is licensed under the Creative Commons Attribution International License (CC BY 4.0).

http://creativecommons.org/licenses/by/4.0/

\begin{abstract}
Drought stress at the reproductive stage causes severe damage to productivity of wheat. However, little is known about the metabolites associated with drought tolerance. The objectives of this study were to elucidate changes in metabolite levels in wheat under drought, and to identify potential metabolites associated with drought stress through untargeted metabolomic profiling using a liquid chromatography-high resolution mass spectrometry (LC-HRMS)-based technique called Isotopic Ratio Outlier Analysis. Metabolomic analysis was performed on flag leaves of drought-stressed and control (well-watered) plants after 18 days of post-anthesis drought stress at three-hour intervals over a 24-hour period. Out of 723 peaks detected in leaves, 221 were identified as known metabolites. Sixty known metabolites were identified as important metabolites by 3 different methods, PLS-DA, RF and SAM. The most pronounced accumulation due to drought stress was demonstrated by tryptophan, proline, pipecolate and linamarin, whereas the most pronounced decrease was demonstrated by serine, trehalose, N-acetyl-glutamic acid, DIBOA-glucoside etc. Three different patterns of metabolite accumulation were observed over 24-hour period. The increased accumulated metabolites remained higher during all 8 time points in drought stressed leaves. On the contrary, metabolites that showed decreased level remained significantly lower during all or the most time points. However, the levels of some decreased metabolites were lower during the day, but higher during night in drought stressed leaves. Both univariate and multivariate analyses predicted that
\end{abstract}

${ }^{\star}$ Co-first authors. 
$\mathrm{N}$-acetyl-glutamic acid, proline, pipecolate, linamarin, tryptophan, and DIBOA-glucoside could be potential metabolite biomarkers, and their levels could serve as indicators of drought tolerance in wheat.

\section{Keywords}

Drought, Post-Anthesis, Wheat, Metabolites, LC-MS

\section{Introduction}

Wheat is one of the most widely produced and consumed cereal grains worldwide and global wheat demand is expected to rise in excess of 880 million metric tons by 2050 [1]. Production of major crops, including wheat, is declining in some regions of the world due to climate change, especially progressive drought stress, induced by either declining rainfall or higher water stress allied with a hot climate [2] [3] [4]. Water stress accompanied by other abiotic stresses can decrease potential crop yield by $>50 \%$ [5]. Water stress during the flowering stage and onwards in wheat typically interrupts photosynthesis and increases translocation of carbohydrates from source to sink, reduces grain number, weight and yield, accelerates phasic development, reduces grain filling duration and enhances senescence [6] [7] [8] [9] [10]. Genetic improvement is the most effective and sustainable method to reduce the detrimental effect of water stress. However, the genetic improvement of traits that confer stress tolerance can only be achieved when we have a better understanding of the biochemical mechanism controlling those traits. The analyses of biologically important molecules are necessary to understand which molecules are influencing stress tolerance mechanisms in wheat plants. Under water-stress, plants produce an array of biomolecules including different metabolites [11]. Plants can modify their physiology to acclimate to abiotic conditions through metabolic changes [12] [13]. Changes in the metabolic level in an organism are likely correlated with the phenotype, as metabolites are the end-products of biological systems [14]. Other than the changes in the level of metabolites, little is known about their complex metabolic regulation under water stress or potential role in developing climate resilient wheat.

Crop yield has shown significant correlation with metabolites under drought [15]. Metabolic composition is highly variable in a wide variety of plants including major crops like wheat [16], rice [17], maize [15], and cassava [14]. These metabolic changes in plants have also occurred due to progressive water deficit conditions accompanied by lower leaf water potentials [18]. Water stress induces metabolic changes and accumulation of metabolites including proline, ascorbic acid, glutathione, phenolics, and detoxifying enzymes in cereals [19] [20]. Bowne et al. (2012) studied metabolic responses in wheat grown under contrasting water levels (drought and irrigated conditions) and reported that amino acids, most notably proline, tryptophan, and the branched chain amino acids, including leu- 
cine, isoleucine, and valine exhibited higher levels of expression in the leaves of drought grown plants [21]. Additionally, different metabolites including phenolic compounds, amino acids, fatty acids, organic acids, carbohydrates, and sterol-based compounds can be altered depending on the phenology and physiological status of the plant under stress [22]. Stress tolerance is a polygenic trait that needs to be incorporated in wheat to develop climate resilient germplasm. Quantitative trait loci can be identified and tagged on genomic regions in a plant using suitable biomarkers through application of molecular breeding techniques. If a set of water stress related metabolic biomarkers can be identified for wheat, they could be used as targeted, fast, and low-cost diagnostic tools to select superior performing lines in breeding programs.

Metabolic profiling allows for comprehensive analyses of a range of metabolites that have great value in both phenotyping and plant diagnostics [23]. Recent progress in mass spectrometry with advanced data processing technology allows simultaneous measurement of hundreds of chemically different metabolites and investigates more thoroughly the regulation of metabolic networks to study their influence on complex traits. Such metabolic profiling can meaningfully contribute to the study of stress biology in wheat and could offer a set of drought related metabolite biomarkers. To date, there are insufficient biomarkers available to adequately screen cereal crops for drought tolerance or susceptibility. The objectives of this study were: 1 ) to elucidate the differential metabolite accumulation in wheat leaves under post-anthesis drought stress conditions over 24 hours time period, and the involvement of those metabolites in different pathways in relation to drought tolerance and 2) to identify potential metabolite biomarkers associated with drought stress in wheat. To achieve this objective, we employed a non-targeted LC-HRMS Isotopic Ratio Outlier Analysis (IROA) Global Metabolomics method [24] for identifying metabolites from the leaf tissue of the drought stressed and control wheat plants at 8 time points of 24 -hour period.

\section{Materials and Methods}

\subsection{Plant Material and Growth Conditions}

The soft winter wheat variety 'SS8641' (GA-881130/2*GA-881582) is widely grown in the southeastern USA, developed by the University of Georgia Wheat Breeding Program, and was subjected to post-anthesis drought stress and LC-HRMS based untargeted metabolic profiling in this study. The SS8641 is a mid-maturing, high yielding wheat variety and has high test weight with good straw strength. It showed resistance to Hessian fly biotypes B and E, and possesses powdery mildew genes $\operatorname{Pm} 1$ and rust resistance genes $\operatorname{Lr} 37 / \mathrm{Yr} 17 / \operatorname{Sr} 38$. Since the wheat flag leaf is a vital source of energy assimilates during grain filling [25] [26], we initially screened a collection of soft wheat germplasm (developed by different US public wheat breeding programs) for thylakoid membrane (indirect assessment of Photosystem II damage) and chlorophyll damage under water stress at flag leaf. The SS8641 wheat genotype showed minimal thylakoid 
membrane and chlorophyll damage under long-term water stress. This finding led to further investigation of metabolites accumulated during grain filling under drought stress in SS8641.

Initially, seeds of SS8641 were exposed to vernalization at $4^{\circ} \mathrm{C}$ for 6 weeks to induce flowering. After vernalization, three germinated seeds were planted in each pot and kept in an environmentally controlled greenhouse located at the campus of the University of Florida in Gainesville. The greenhouse was maintained at day/night temperature of $20 / 15^{\circ} \mathrm{C} \pm 0.5^{\circ} \mathrm{C}(16 \mathrm{~h}$ daylight and $8 \mathrm{~h}$ night time) with a relative humidity of $50 \% \pm 2 \%$. Plants were manually irrigated at three day intervals and maintained at $100 \%$ field capacity (FC) until exposed to drought stress. Plants were also fertilized with two splits of $12 \mathrm{~g}$ of Scott's Osmocote (20-4-8 NPK fertilizer) during the experimental period: one at 7 days and another split at 45 days after transplanting. Once plants reached the flowering stage, pots were randomly assigned to two different watering regimes with three replications as follows: control treatment (well-watered, 100\% FC) and drought treatment (water-deficit, 25\% FC). To determine FC, the weights of pot plus dried soil weight were recorded as average $2.24 \mathrm{~kg}$, then pots were irrigated to $100 \% \mathrm{FC}$ and average weight increased to $3.44 \mathrm{~kg}$ (moisture content was assessed as $1.20 \mathrm{~kg} / \mathrm{pot})$. Pots in the control treatment were maintained at $100 \%$ FC, while drought treatments were maintained up to $25 \%$ of the FC. After 18 days of the drought treatment, plants were characterized for photosystem and chlorophyll damages, and flag leaves were collected for metabolic profiling.

\subsection{Physiological Characterization}

Chlorophyll fluorescence (the ratio of variable, Fv, to maximum fluorescence, Fm) and SPAD chlorophyll content were used as indirect methods to assess thylakoid membrane [27] [28] and chlorophyll damages due to stress following the methods described by Talukder et al. (2014) [29]. As photosystem II is housed at the thylakoid membrane, damage to thylakoid membrane is considered as an indicator of damage to photosystem II under stress condition. The lower ratio of $\mathrm{Fv} / \mathrm{Fm}$ indicates more damage to Photosystem II due to stress. Measurements of $\mathrm{Fv} / \mathrm{Fm}$ were taken on the abaxial surface of intact flag leaves at one third of the length from the ligule after $30 \mathrm{~min}$ of dark adaptation on three flag leaves of each plant (biological replication). Fluorescence was measured using a pulse modular fluorometer (Model OS5-FL, Opti-Sciences, Hudson, NH, USA) in both the control and drought stressed leaves at 18 days after flowering. A self-calibrating SPAD chlorophyll meter (Model 502, Spectrum Technologies, Plainfield, IL) was used to measure chlorophyll content on the same flag leaves and leaf blade areas where chlorophyll fluorescence measurements were taken. In each treatment (control and drought), chlorophyll content was measured from three flag leaves (3 different plants) and the average of three measurements was used to represent chlorophyll content. The lower value of SPAD chlorophyll content indicates more damage to chlorophyll due to stress. 
Plasma membrane damage (PMD) was measured using the method described by Ristic and Cass (1993) [30]. Leaf disks (diameter $=5 \mathrm{~mm}$ ) were collected from six individual flag leaves within each biological replication after the drought treatment and placed in de-ionized water $(24 \mathrm{ml})$ in sealed vials. The vials were shaken overnight on a shaker at $5^{\circ} \mathrm{C}$. Electrical conductivity $(\mu \mathrm{S} / \mathrm{cm})$ of the aqueous solution was measured with a Metter Toledo (Seven-Multi S70) conductivity meter. The tissue samples were then autoclaved for $20 \mathrm{~min}$. The conductivity of the solution was again measured after shaking the samples overnight at $5^{\circ} \mathrm{C}$. The percent electrolyte leakage was calculated based on the conductivity before and after autoclaving. The average value of six flag leaves within each biological replication was used to estimate \%PMD according to the formula, $\left[\left(\% \mathrm{~L}_{\mathrm{D}}-\% \mathrm{~L}_{\mathrm{C}}\right) /\left(\mathrm{X}-\% \mathrm{~L}_{\mathrm{C}}\right)\right] \times 100$, where $\mathrm{L}_{\mathrm{D}}$ and $\mathrm{L}_{\mathrm{C}}$ are electrolyte leakages under drought stress and control conditions respectively, and $\mathrm{X}$ is assumed to be $100 \%$ leakage corresponding to $100 \%$ membrane damage.

\subsection{Measurements of Growth and Yield}

Plants were harvested after physiological maturity and oven-dried at $60^{\circ} \mathrm{C}$ for five days. After drying, total biomass was measured per plant. The spikes were hand threshed and the chaff was cleaned off. Grain weight was measured and the harvest index was calculated by dividing grain weight/total biomass. Grain yield per spike was calculated by dividing total grain weight by spike number. Grain number per spike was calculated by dividing total grain number by the number of spikes for that plant. Two hundred grains were randomly selected and weighed, and converted to 1000-grain weight.

\subsection{Leaf Tissue Collection and Sample Preparation for Metabolomics}

Flag leaves were collected in 3-hour increments (06:00 AM; 09:00 AM; 12:00 noon; 15:00 PM; 18:00 PM, 21:00 PM, 24:00 PM and 03:00 AM) over a 24 hour time period. Collected flag leaf from each pot was considered a biological replication. A triplicate samples were collected at each sampling time point as our experiment was maintained in a well environmentally controlled greenhouse and experimental treatments showed high accuracy within replication. Sampled leaf tissues were frozen in liquid nitrogen immediately after collection and then stored at $-80^{\circ} \mathrm{C}$ until processing. Leaf tissue samples were lyophilized and ground using a tissuelyser (24 samples per treatment for a total of 48 samples). For metabolomic analysis, $5 \mathrm{mg}$ of experimental material was weighed and added to $5 \mathrm{mg}$ of freshly ground wheat internal standard (IS). The IS was an isotopically labeled wheat leaf that had been grown in an atmosphere of ${ }^{13} \mathrm{C}$ labeled carbon dioxide resulting in a uniform and universal labeling of approximately 97\% (IROA Technologies) [24]. Next, $500 \mu \mathrm{l}$ of methanol/10mM aqueous ammonium acetate (50:50) was added to the dried powder and vortexed for $1 \mathrm{~min}$ at room temperature. The resulting mixture was further sonicated for $20 \mathrm{~min}$, 
and centrifuged at $17,000 \mathrm{G}$ for 10 minutes at $4^{\circ} \mathrm{C}$. A $350 \mu \mathrm{l}$ aliquot of the supernatant was transferred to a clean dry eppendorf tube, and dried under a gentle nitrogen gas stream (at $30^{\circ} \mathrm{C}$ ). The dried sample was reconstituted in $100 \mu \mathrm{l}$ of $0.1 \%$ aqueous format.

\subsection{Untargeted Metabolomics}

The reconstituted samples were analyzed for untargeted metabolites on a Liquid Chromatography High Resolution Mass Spectrometery (LC-HRMS) platform. Untargeted metabolomics profiling was performed on a Thermo Q-Exactive Oribtrap mass spectrometer with Dionex UHPLC and autosampler. All samples were analyzed in positive and negative modes with heated electrospray ionization with a mass resolution of 35,000 at $\mathrm{m} / z$ 200. Chromatographic separation was achieved on an ACE 18 -pfp $100 \times 2.1 \mathrm{~mm}, 2 \mu \mathrm{m}$ column with mobile phase A as $0.1 \%$ formic acid in water and mobile phase B as acetonitrile. The flow rate was $350 \mu \mathrm{L} / \mathrm{min}$ and column temperature was $25^{\circ} \mathrm{C}$. Total run time per sample was 21 minutes. Quality assurance and quality control (QA/QC) guidelines were followed during untargeted profiling of assays with the addition of stable-isotopic internal standards to evaluate reproducibility, injection standards, and the repeated analysis of a large pooled plasma sample. Injection reproducibility was typically less than $10 \%$ even without a ratio to an internal standard. The native Thermo ".raw" output files were converted to .mzXML files using ProteoWizard (Version 2).

\subsection{Metabolic Data Processing}

One of the greatest challenges of most metabolic profiling experiments is the ability to differentiate peaks of biological origin from artifact peaks, and to accurately identify and quantitate the peaks of interest. Since we used an IROA-labeled plant material as our internal standard this study followed the IROA "Phenotypic" global labeling and bioinformatics protocols in which the Internal Standard (IS) is labeled at $95 \%{ }^{13} \mathrm{C}$. Therefore, all biological compounds are paired natural abundance (NA) and IS, and each pair carries distinct molecular signatures. Molecules can be distinguished from each sample set, as they have differing masses [31] [32].

For IROA, control and drought treated samples were analyzed as a single composite sample by LC-MS. Algorithms pair identified biological peaks, and unlabeled NA artifacts were identified and discarded. All biological compounds had two paired peaks; the peak from the ${ }^{12} \mathrm{C}$-media is mirrored by a second peak from the ${ }^{13} \mathrm{C}$-media. The distance between the monoisotopic peaks readily identified the number of carbons in the compound. The corresponding $\mathrm{M}^{+1}$ and $\mathrm{M}^{-1}$ peaks (and $\mathrm{M}^{+2}$ and $\mathrm{M}^{-2}$ etc. peaks) which are a mass difference of $1.00335 \mathrm{amu}$ (mass difference between a ${ }^{12} \mathrm{C}$ and ${ }^{13} \mathrm{C}$ isotope), gave the IROA peaks a characteristic U-shape "smile" pattern. Accurate mass together with the knowledge of the number of carbons in a molecule greatly facilitated metabolite identification. 
The ClusterFinder program was used to identify, align and quantitate the IROA peaks in the .mzXML files. The files were scanned for IROA peaks down to an intensity level of 1 million in both positive and negative modes with an assumed maximum of $10 \mathrm{ppm}$ mass error. Settings for the experimental samples in the nontargeted analysis assumed a natural abundance (1.1\%) isotopic balance while the IS was assumed to have a $97 \%$ isotopic balance. The resulting list of IROA peaks was manually curated to define 394 compounds that were seen in both the IS and in the experimental samples. Once the physical attributes for the 494 compounds were identified, a targeted analysis for all of these compounds was imposed on every sample. This resulted in a non-sparse dataset, i.e. there was a value for every compound for every sample and the dataset was exported for analysis. The metabolites were annotated by searching against an in-house metabolite database, Mass Spectrometry Metabolite Library of Standards (MSMLS) (http://iroa.com/page/Mass\%20Spectrometry\%20Metabolite\%20Library\%20of\% 20Standards).

\subsection{Metabolic Data Analysis}

Data tables with metabolite peaks $(\mathrm{mz} / \mathrm{rt})$ at 8 time points under both drought and control conditions were formatted as comma separated values (.csv) files and uploaded to the MetaboAnalyst 3.0 server (http://www.metaboanalyst.ca) [33]. To shrink any possible variance and to improve the performance for downstream statistical analysis, metabolite data generated by LC-HRMS were checked for data integrity and normalized using Metabo Analyst's normalization protocols (selecting normalization by sum, log transformation and auto-scaling) for statistical analysis.

Univariate analysis (t-test and one way ANOVA) was applied to calculate the statistical significance and fold change of the metabolites between two group means (drought over control). As the multivariate methods take all the variables into consideration, we applied multivariate methods for comprehensive data analysis e.g. supervised methods- Partial Least Squares Discriminant Analysis (PLS-DA), Random Forest (RF) classification, and unsupervised method-Hierarchical clustering with heatmap. The supervised method, PLS-DA was used to maximize the difference of metabolic profiles between control and drought groups to enable the detection of metabolites exist in the biological samples. A heat map was generated based on the Pearson distance measure and the Ward clustering algorithm, showing top 60 metabolites selected by PLS-DA VIP (variable importance in projection) score using a significance level of $\mathrm{P} \leq$ 0.05 , and post-hoc analysis of Fisher's LSD. The samples were arranged according to their sampling time points in both control and drought groups. The important metabolites were identified by using 3 different methods separately: SAM (Significant Analysis of Metabolites), PLS-DA and RF.

The pathway analysis was performed using Metabo Analyst for the identified important metabolites using Oryza sativa japonica and Arabidopsis thaliana 
pathway libraries. The Kyoto Encyclopedia of Genes and Genomes (KEGG) pathway database (http://www.genome.ad.jp/kegg/pathway.html) was also used for the metabolites that were not found in the rice and Arabidopsis pathway libraries. To identify the potential biomarkers associated with drought stress, the Receiver Operating Characteristic (ROC) curve based 'Biomarker Analysis' module of the MetaboAnalyst was applied. Both classical univariate ROC curve analysis and multivariate ROC curve exploratory analysis were used to identify the promising biomarkers with high sensitivity and high specificity.

\section{Results}

\subsection{Physiological Traits}

We measured physiological traits to assess the impact of the drought stress 18 days after stress initiation at anthesis. The chlorophyll content (SPAD value), maximum quantum efficiency of PSII (Fv/Fm) and electrolyte leakage (\%) were determined on flag leaves for both control (well-watered) and drought conditions (Table 1). Drought stress caused a significant reduction $(\sim 11 \% ; \mathrm{p}<0.05)$ in chlorophyll content of wheat flag leaves compared to control. The photosynthetic apparatus (PSII) was damaged to some extent which was apparent from the reduced $\mathrm{Fv} / \mathrm{Fm}$ value under drought condition (Table 1), although the reduction was not statistically significant. Electrolyte leakage, in contrast, increased substantially $(\sim 18 \%)$ under drought condition compared to control resulting in $\sim 30 \%$ damage to plasma membranes of the drought stressed plants.

\subsection{Morphological Traits}

Total shoot biomass at maturity, grain weight/plant, grain weight/spike, 1000-grain weight, grains/spike, and harvest index showed significant reduction under drought stress compared to control condition as expected (Table 1).

Table 1. Physiological and morphological traits of SS8641 wheat line under post-anthesis drought stress and control conditions.

\begin{tabular}{lcc}
\hline Traits & Control (Mean \pm SE) & Drought (Mean \pm SE) \\
\hline SPAD chlorophyll content & $60.1 \pm 0.8$ & $53.4 \pm 1.8$ \\
Chlorophyll a florescence (Fv/Fm) & $0.781 \pm 0.003$ & $0.746 \pm 0.017$ \\
Electrolyte leakage (\%) & $38.4 \pm 0.7$ & $57.1 \pm 0.7$ \\
Plasma membrane damage (PMD \%) & - & $30.4 \pm 0.5$ \\
Total shoot dry biomass at maturity (g/plant) & $16.0 \pm 2.1$ & $10.4 \pm 0.1$ \\
Grain weight/plant & $7.5 \pm 0.6$ & $5.1 \pm 0.1$ \\
1000 grain weight (g) & $49.4 \pm 0.3$ & $41.0 \pm 1.5$ \\
Number of grains/spike & $27.8 \pm 1.7$ & $24.1 \pm 1.5$ \\
Grain weight/spike (g) & $1.8 \pm 0.1$ & $1.3 \pm 0.1$ \\
Harvest Index & $44.4 \pm 1.9$ & $40.0 \pm 0.4$
\end{tabular}

$\mathrm{SE}=$ Standard Error. 


\subsection{Profiling of Leaf Metabolites}

Metabolite profiling by LC-HRMS detected a total of 723 peaks from wheat flag leaves. Among the detected peaks, 221 were identified as known metabolites (266 including duplications) and the remaining peaks were unknown metabolites. The identified compounds included amino acids, sugars, organic acids, organic compounds, polyamines, fatty acids, nucleosides/nucleobases and other compounds.

The supervised clustering method, Partial Least Squares-Discriminant Analysis (PLS-DA) was performed for two conditions at 8 different time points. Five PLS-components (PCs) explained $66.5 \%$ of the total variation, with the first and second PCs contributing $45.1 \%$ and $8.5 \%$, respectively (Figure 1). Drought and control samples were clearly separated across the eight different sampling points in the scores plot between PC1 and PC2 (Figure 1) with minimum overlapping within the group. This separation of drought and control samples clearly indicates the altered state of metabolite levels in the wheat leaves under drought condition.

One hundred fifty two metabolites were found significantly different ( $\mathrm{t}$-test, $\mathrm{p}$ $<0.05$ ) between drought and control conditions (Supplemental Table S1). Of these, volcano plot analysis identified 112 known metabolites which had a fold change $\geq 1.5$ (drought/control, either positive or negative) in drought treated plants compared to controls. Among different metabolite groups, accumulation of a number of amino acids significantly increased in the drought treated leaves compared to control, including tryptophan (7.1 fold), proline (3.9 fold), phenylalanine (1.8 fold), tyrosine (1.6 fold) and isoleucine ( 1.5 fold). However, the level of some amino acids and their derivatives, including serine ( -2.4 fold), glutathione ( -2.3 fold), glutamine ( -1.9 fold), 4-Aminobutanoate (GABA, -1.9 fold), and threonine ( -1.9 fold) decreased under drought stress (Supplemental Table $\mathrm{S} 1)$. The levels of sugars and their derivatives, including sucrose ( -2.0 fold), rafffinose $(-2.0$ fold $)$, trehalose $(-2.1$ fold $)$, sedoheptulose $(-1.7$ fold $)$, 6-( $\alpha$-D-glucosaminyl)-1D-myo-inositol ((GlcN)1 (Ino)1, -2.9 fold), xylonate $(-2.1$ fold) decreased in flag leaves of drought induced plants compared to control (Supplemental Table S1). A large number of organic compounds and organic acids including those involved in the Krebs cycle (for example, pyruvate, phosphonatoenolpyruvate, alpha-ketoglutaric acid) were also reduced under drought stress with the exception of phenyl pyruvate ( 1.7 fold), pipecolate $(2.2$ fold), and linamarin (3.3 fold). In addition, fatty acids (e.g. linoleate, palmitoleic acid etc.), amines (spermidine, piperidine), nucleosides (adenine, guanosine) and other metabolites (indoline, sesbanimide, aminoimidazoleribotide, carbetamide, pyridoxal, etc.) were also differentially accumulated under drought stress (Supplemental Table S1).

Significantly different metabolites were analyzed by hierarchical clustering with heat map in order to visualize the effect of drought stress over the control at eight different time points (Figure 2). The heatmap formed two major clusters 

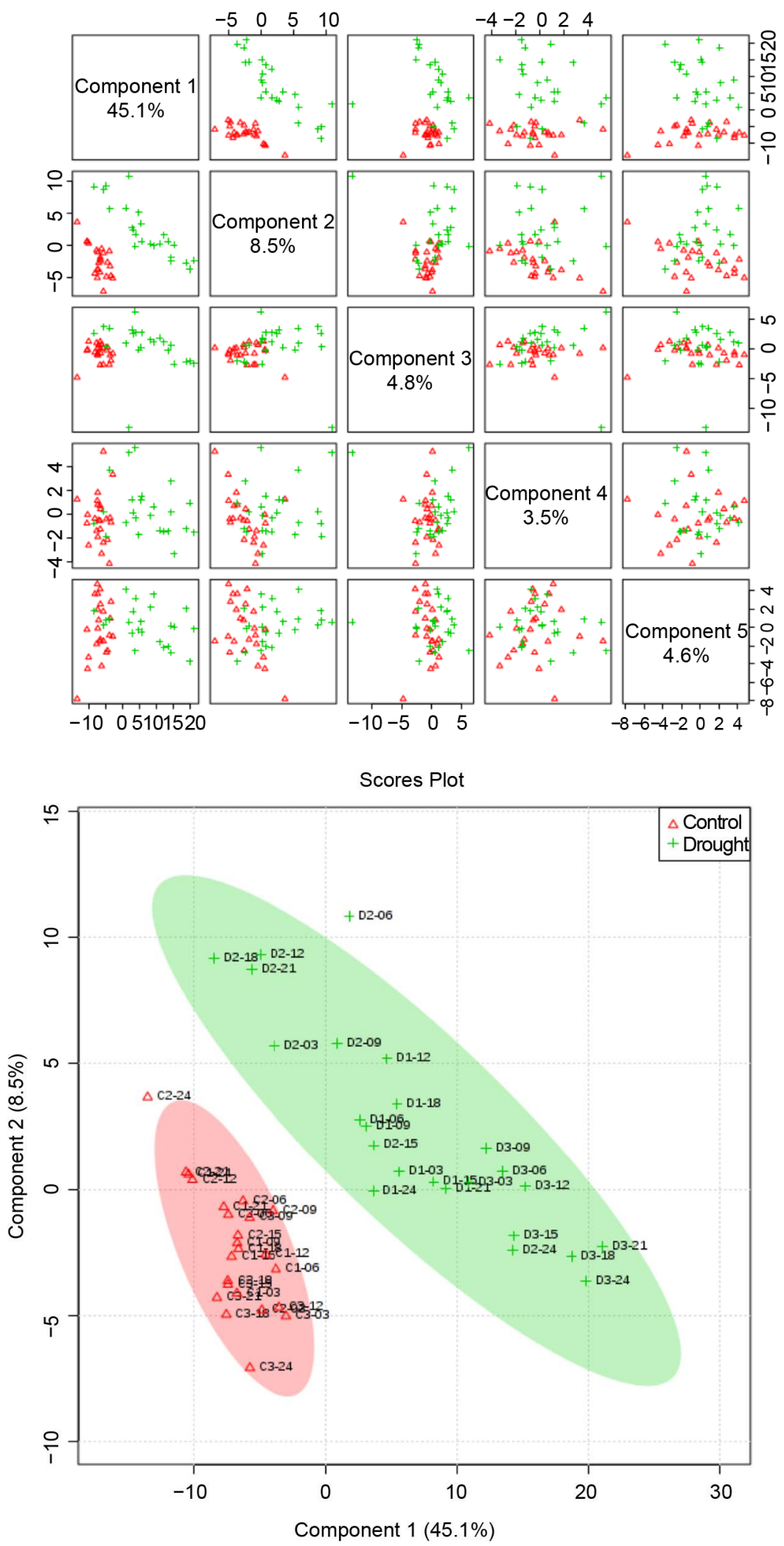

Figure 1. Partial least square discriminant analysis (PLS-DA) and 2D Scores plot for the wheat SS8641 leaves over the $24 \mathrm{~h}$ time period (8 time points) under control (irrigated) and drought conditions. Samples under control and drought condition at different time points did not overlap with each other (although overlapped within group), indicating an altered state of metabolite levels in the wheat leaves. 


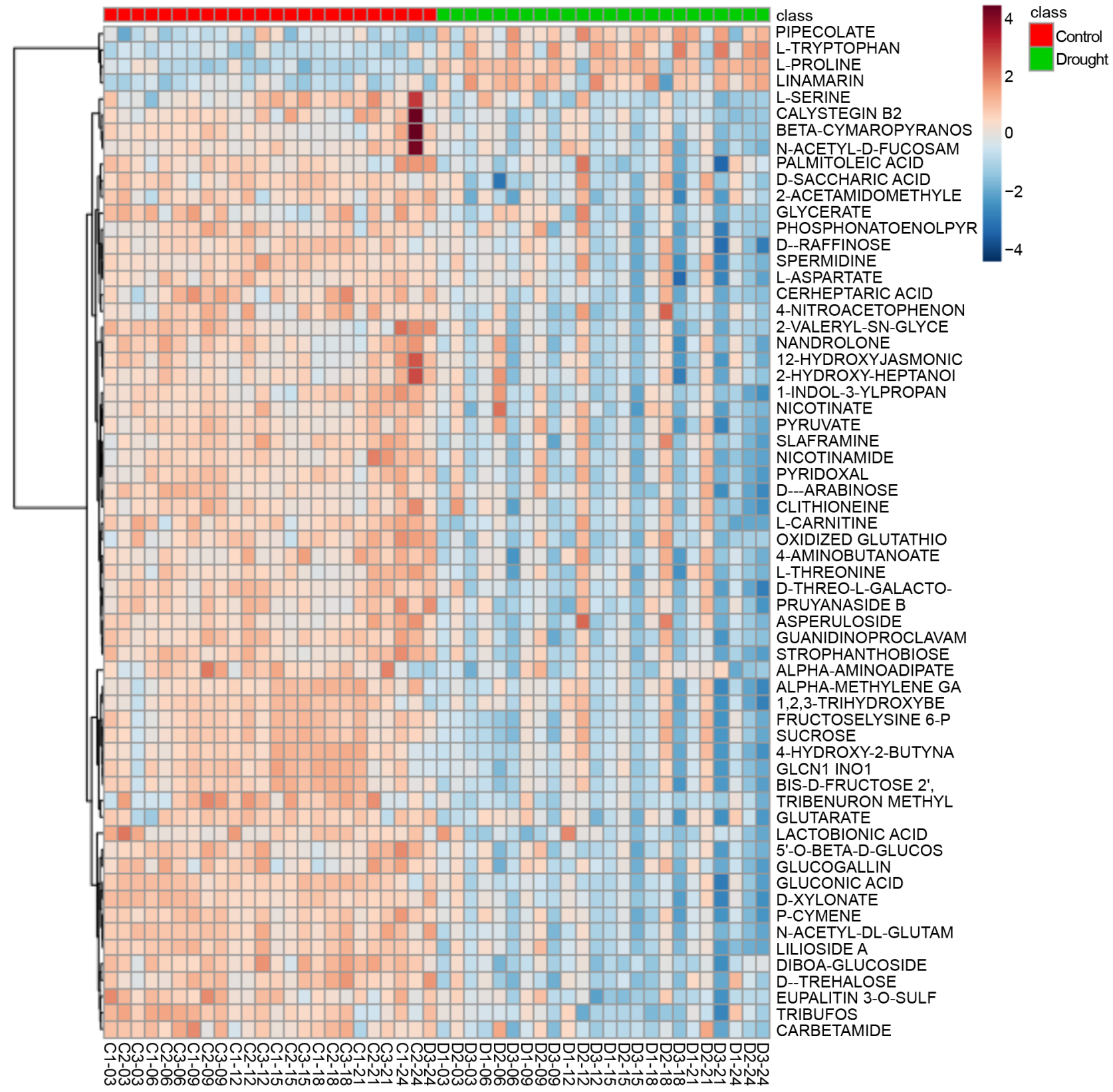

Figure 2. Heatmap showing levels of top metabolites based on partial least square discriminant analysis (P LS-DA) VIP scores at 8 different time points under control and drought conditions. The heatmap was generated using "Pearson" for distance measure and "Ward" for clustering algorithm. The hierarchical clustering was performed only on the metabolites and the samples were arranged based on their time points in two groups.

with opposite pattern of metabolite accumulation. The first cluster was represented by metabolites that accumulated to high levels under drought stress, including tryptophan, proline, linamarin and pipcolate. The second cluster included metabolites that decreased $\geq 1.5$ fold under drought compared to control, including serine, 4-Aminobutanoate (GABA), oxidized glutathione, sucrose, DIBOA-glucoside, raffinose, arabinose, N-acetyl-DL-glutamic acid, Pyruvate, glycerate, nicotinate, tribenuron methyl, palmitoleic acid, nandrolone, calystegin 
B2, slaframine, pyridoxal and others. The clustering of metabolites in two groups clearly indicates the metabolic changes in flag leaves under stress condition.

A comparison of statistical models was carried out to identify the important metabolites associated with drought condition using: SAM, PLS-DA and RF (Table 2). The SAM plot identified 61 significantly different compounds with

Table 2. Important metabolites with their identifier number (KEGG ID/PubChemCID $\left(^{*}\right) / \mathrm{UNDP}$ ID), molecular formula (MF), identified through Partial Least Square Discrepant Analysis (PLS-DA), Random Forest (RF) and Significant Analysis of Metabolites (SAM). (KEGG Kyoto Encyclopedia of Genes and Genomes, UNDP = United Natural Products Database).

\begin{tabular}{|c|c|c|c|c|c|c|}
\hline Name of metabolites & Compound ID & $\begin{array}{l}\text { Molecular } \\
\text { Formula }\end{array}$ & Compound type & $\begin{array}{c}\text { SAM } \\
\text { (d.value) }\end{array}$ & $\begin{array}{c}\text { RF (Mean } \\
\text { Decrease Accuracy) }\end{array}$ & $\begin{array}{c}\text { PLS-DA } \\
* *(\text { VIP score })\end{array}$ \\
\hline L-Proline & $\mathrm{C} 00148$ & $\mathrm{C}_{5} \mathrm{H}_{9} \mathrm{NO}_{2}$ & Amino acid & 3.034 & 0.0180 & 1.369 \\
\hline L-Tryptophan & $\mathrm{C} 00078$ & $\mathrm{C}_{11} \mathrm{H}_{12} \mathrm{~N}_{2} \mathrm{O}_{2}$ & Amino acid & 2.745 & 0.0024 & 1.284 \\
\hline L-Aspartate & C00049 & $\mathrm{C}_{4} \mathrm{H}_{7} \mathrm{NO}_{4}$ & Amino acid & -2.017 & 0.0016 & 1.099 \\
\hline L-Serine & $\mathrm{C} 00065$ & $\mathrm{C}_{3} \mathrm{H}_{7} \mathrm{NO}_{3}$ & Amino acid & -1.978 & 0.0012 & 1.078 \\
\hline L-Threonine & $\mathrm{C} 00188$ & $\mathrm{C}_{4} \mathrm{H}_{9} \mathrm{NO}_{3}$ & Amino acid & -1.965 & 0.0023 & 1.091 \\
\hline 4-Aminobutanoate (GABA) & $\mathrm{C} 00334$ & $\mathrm{C}_{4} \mathrm{H}_{9} \mathrm{NO}_{2}$ & Amino acid & -2.184 & 0.0045 & 1.151 \\
\hline L-Carnitine & $\mathrm{C} 00318$ & $\mathrm{C}_{7} \mathrm{H}_{15} \mathrm{NO}_{3}$ & $\begin{array}{l}\text { Amino acid } \\
\text { derivative }\end{array}$ & -2.609 & 0.0051 & 1.336 \\
\hline Clithioneine & UNPD 118571 & $\mathrm{C}_{13} \mathrm{H}_{22} \mathrm{~N}_{4} \mathrm{O}_{5} \mathrm{~S}$ & $\begin{array}{l}\text { Amino acid } \\
\text { derivative }\end{array}$ & -2.037 & 0.0019 & 1.136 \\
\hline D-(+)-Trehalose & $\mathrm{C} 01083$ & $\mathrm{C}_{12} \mathrm{H}_{22} \mathrm{O}_{11}$ & Sugar & -2.827 & 0.0053 & 1.376 \\
\hline Sucrose & $\mathrm{C} 00089$ & $\mathrm{C}_{12} \mathrm{H}_{22} \mathrm{O}_{11}$ & Sugar & -2.573 & 0.0028 & 1.268 \\
\hline D-Threo-L-galacto-octose & $16019992^{*}$ & $\mathrm{C}_{8} \mathrm{H}_{16} \mathrm{O}_{8}$ & Sugar & -2.513 & 0.0065 & 1.299 \\
\hline D-(-)-Arabinose & $\mathrm{C} 00216$ & $\mathrm{C}_{5} \mathrm{H}_{10} \mathrm{O}_{5}$ & Sugar & -2.188 & 0.0066 & 1.202 \\
\hline D-(+)-Raffinose & C00492 & $\mathrm{C}_{18} \mathrm{H}_{32} \mathrm{O}_{16}$ & Sugar & -2.071 & - & 1.103 \\
\hline N-Acetyl-D-Fucosamine & $\mathrm{C} 15480$ & $\mathrm{C}_{8} \mathrm{H}_{15} \mathrm{NO}_{5}$ & Sugar & -1.892 & 0.0027 & 1.053 \\
\hline $\begin{array}{l}\text { 6-( } \alpha \text {-D-glucosaminyl)-1D- } \\
\text { myo-inositol, (GlcN)1 (Ino)1 }\end{array}$ & C15658 & $\mathrm{C}_{12} \mathrm{H}_{23} \mathrm{NO}_{10}$ & Sugar alcohol & -2.775 & 0.0033 & 1.331 \\
\hline $\begin{array}{l}\text { Bis-D-fructose 2',1:2,1' } \\
\text {-dianhydride }\end{array}$ & $\mathrm{C} 04333$ & $\mathrm{C}_{12} \mathrm{H}_{20} \mathrm{O}_{10}$ & Sugar dianhydride & -2.720 & 0.0064 & 1.313 \\
\hline D-xylonate & $\mathrm{C} 00502$ & $\mathrm{C}_{5} \mathrm{H}_{10} \mathrm{O}_{6}$ & Sugar acid & -3.090 & 0.0204 & 1.469 \\
\hline $\begin{array}{l}\text { alpha-Methylene gamma } \\
\text {-butyrolactone }\end{array}$ & $68352^{*}$ & $\mathrm{C}_{5} \mathrm{H}_{6} \mathrm{O}_{2}$ & Sugar acid & -2.114 & - & 1.120 \\
\hline Spermidine & $\mathrm{C} 00315$ & $\mathrm{C}_{7} \mathrm{H}_{19} \mathrm{~N}_{3}$ & Amine & -2.106 & 0.0078 & 1.130 \\
\hline Palmitoleic acid & $\mathrm{C} 08362$ & $\mathrm{C}_{16} \mathrm{H}_{30} \mathrm{O}_{2}$ & Fatty acid & -1.949 & 0.0015 & 1.062 \\
\hline N-Acetyl-DL-glutamic acid & $\mathrm{C} 00624$ & $\mathrm{C}_{7} \mathrm{H}_{11} \mathrm{NO}_{5}$ & Organic acid & -3.467 & 0.0267 & 1.568 \\
\hline Guanidinoproclavaminic acid & $\mathrm{C} 06657$ & $\mathrm{C}_{9} \mathrm{H}_{16} \mathrm{~N}_{4} \mathrm{O}_{4}$ & Organic acid & -2.892 & 0.0064 & 1.395 \\
\hline Gluconic acid & C00257 & $\mathrm{C}_{6} \mathrm{H}_{12} \mathrm{O}_{7}$ & Organic acid & -3.163 & 0.0092 & 1.468 \\
\hline Cerheptaric acid & UNPD64017 & $\mathrm{C}_{7} \mathrm{H}_{12} \mathrm{O}_{8}$ & Organic acid & -2.576 & 0.0029 & 1.292 \\
\hline Pipecolate & $\mathrm{C} 00408$ & $\mathrm{C}_{6} \mathrm{H}_{11} \mathrm{NO}_{2}$ & Organic acid & 2.442 & - & 1.121 \\
\hline Lactobionic acid & $7314^{*}$ & $\mathrm{C}_{12} \mathrm{H}_{22} \mathrm{O}_{12}$ & Organic acid & -2.228 & 0.0029 & 1.203 \\
\hline
\end{tabular}




\section{Continued}

\begin{tabular}{|c|c|c|c|c|c|c|}
\hline 12-Hydroxyjasmonic acid & C21385 & $\mathrm{C} 12 \mathrm{H} 18 \mathrm{O} 4$ & Organic acid & -2.170 & 0.0019 & 1.161 \\
\hline D-Saccharic acid & C00818 & C6H10O8 & Organic acid & -2.170 & 0.0025 & 1.119 \\
\hline Glutarate & C00489 & $\mathrm{C}_{5} \mathrm{H}_{8} \mathrm{O}_{4}$ & Organic acid & -2.009 & 0.0051 & 1.076 \\
\hline alpha-Aminoadipate & C00956 & $\mathrm{C}_{6} \mathrm{H}_{11} \mathrm{NO}_{4}$ & Organic acid & -2.004 & 0.0012 & 1.135 \\
\hline Phosphonatoenolpyruvate & C00074 & $\mathrm{C}_{3} \mathrm{H}_{2} \mathrm{O}_{6} \mathrm{P}$ & Organic acid & -1.930 & - & 1.064 \\
\hline Nicotinate & C00253 & $\mathrm{C}_{6} \mathrm{H}_{5} \mathrm{NO}_{2}$ & Organic acid & -1.896 & 0.0026 & 1.110 \\
\hline Glycerate & C00258 & $\mathrm{C}_{3} \mathrm{H}_{6} \mathrm{O}_{4}$ & Organic acid & -1.892 & - & 1.043 \\
\hline Pyruvate & C00022 & $\mathrm{C}_{3} \mathrm{H}_{4} \mathrm{O}_{3}$ & Organic acid & -1.890 & 0.0019 & 1.083 \\
\hline DIBOA-glucoside & C15772 & $\mathrm{C}_{14} \mathrm{H}_{17} \mathrm{NO}_{9}$ & Organic compound & -3.455 & 0.0126 & 1.529 \\
\hline Lilioside A & $101277416^{*}$ & $\mathrm{C}_{11} \mathrm{H}_{20} \mathrm{O}_{9}$ & Organic compound & -3.047 & 0.0119 & 1.472 \\
\hline Tribufos & $5125^{*}$ & $\mathrm{C}_{12} \mathrm{H}_{27} \mathrm{OPS}_{3}$ & Organic compound & -2.784 & 0.0079 & 1.405 \\
\hline P-Cymene & C06575 & $\mathrm{C}_{10} \mathrm{H}_{14}$ & Organic compound & -2.782 & 0.0105 & 1.385 \\
\hline 5'-O-beta-D-Glucosylpyridoxine & C03996 & $\mathrm{C}_{14} \mathrm{H}_{21} \mathrm{NO}_{8}$ & $\begin{array}{l}\text { Organic compound } \\
\text { (Vit B6) }\end{array}$ & -2.751 & 0.0055 & 1.391 \\
\hline Strophanthobiose & $14236734^{*}$ & $\mathrm{C}_{13} \mathrm{H}_{24} \mathrm{O}_{9}$ & Organic compound & -2.706 & 0.0072 & 1.356 \\
\hline Tribenuron methyl & C10962 & $\mathrm{C}_{15} \mathrm{H}_{17} \mathrm{~N}_{5} \mathrm{O}_{6} \mathrm{~S}$ & Organic compound & -2.703 & 0.0036 & 1.318 \\
\hline Eupalitin 3-O-sulfate & $44259774^{*}$ & $\mathrm{C}_{17} \mathrm{H}_{14} \mathrm{O}_{10} \mathrm{~S}$ & Organic compound & -2.590 & 0.0077 & 1.354 \\
\hline Linamarin & C01594 & $\mathrm{C}_{10} \mathrm{H}_{17} \mathrm{NO}_{6}$ & Organic compound & 2.494 & 0.0025 & 1.187 \\
\hline Pruyanaside B & $\begin{array}{l}\text { UNDP } \\
145434\end{array}$ & $\mathrm{C}_{33} \mathrm{H}_{36} \mathrm{O}_{16}$ & $\begin{array}{l}\text { Organic compound } \\
\text { (Phenolicglucoside) }\end{array}$ & -2.430 & 0.0030 & 1.281 \\
\hline Oxidized glutathione & C00127 & $\mathrm{C}_{20} \mathrm{H}_{32} \mathrm{~N}_{6} \mathrm{O}_{12} \mathrm{~S}_{2}$ & $\begin{array}{l}\text { Organic compound } \\
\text { (antioxidant) }\end{array}$ & -2.419 & 0.0017 & 1.240 \\
\hline Slaframine & C06185 & $\mathrm{C}_{12} \mathrm{H}_{20} \mathrm{~N}_{2} \mathrm{O}_{3}$ & Organic compound & -2.331 & 0.0044 & 1.224 \\
\hline Fructoselysine 6-phosphate & C16489 & $\mathrm{C}_{12} \mathrm{H}_{25} \mathrm{~N}_{2} \mathrm{O}_{10} \mathrm{P}$ & $\begin{array}{l}\text { Phosphorylated } \\
\text { compound }\end{array}$ & -2.588 & 0.0036 & 1.274 \\
\hline $\begin{array}{l}\text { 1-(Indol-3-yl)propanol } \\
\text { 3-phosphate }\end{array}$ & C04229 & $\mathrm{C}_{11} \mathrm{H}_{14} \mathrm{NO}_{4} \mathrm{P}$ & $\begin{array}{l}\text { Phosphorylated } \\
\text { compound }\end{array}$ & -2.318 & 0.0020 & 1.242 \\
\hline Nicotinamide & C00153 & $\mathrm{C}_{6} \mathrm{H}_{6} \mathrm{~N}_{2} \mathrm{O}$ & Organic compound & -2.308 & 0.0040 & 1.219 \\
\hline Nandrolone & C07254 & $\mathrm{C}_{18} \mathrm{H}_{26} \mathrm{O}_{2}$ & Organic compound & -2.194 & 0.0024 & 1.194 \\
\hline Glucogallin & C01158 & $\mathrm{C}_{13} \mathrm{H}_{16} \mathrm{O}_{10}$ & Organic compound & -2.147 & 0.0017 & 1.187 \\
\hline Calystegin B2 & C10851 & $\mathrm{C}_{7} \mathrm{H}_{13} \mathrm{NO}_{4}$ & Organic compound & -2.137 & 0.0027 & 1.154 \\
\hline Asperuloside & C09769 & $\mathrm{C}_{18} \mathrm{H}_{22} \mathrm{O}_{11}$ & Organic compound & -2.062 & 0.0093 & 1.101 \\
\hline 1,2,3-Trihydroxybenzene & C01108 & $\mathrm{C}_{6} \mathrm{H}_{6} \mathrm{O}_{3}$ & Organic compound & -2.026 & - & 1.100 \\
\hline 4-Nitroacetophenone & C02803 & $\mathrm{C}_{8} \mathrm{H}_{7} \mathrm{NO}_{3}$ & Organic compound & -1.987 & 0.0013 & 1.052 \\
\hline Beta-Cymaropyranose & C08234 & $\mathrm{C}_{7} \mathrm{H}_{14} \mathrm{O}_{4}$ & Organic compound & -1.968 & - & 1.101 \\
\hline $\begin{array}{l}\text { 2-Valeryl-SN-glycero } \\
\text {-3-phosphocholine }\end{array}$ & $24779499^{*}$ & $\mathrm{C}_{13} \mathrm{H}_{28} \mathrm{NO}_{7} \mathrm{P}$ & Others & -2.457 & 0.0020 & 1.305 \\
\hline Carbetamide & C11075 & $\mathrm{C}_{12} \mathrm{H}_{16} \mathrm{~N}_{2} \mathrm{O}_{3}$ & Others & -2.446 & 0.0015 & 1.264 \\
\hline 4-Hydroxy-2-butynal & C02648 & $\mathrm{C}_{4} \mathrm{H}_{4} \mathrm{O}_{2}$ & Others & -2.384 & 0.0018 & 1.203 \\
\hline Pyridoxal & C00250 & $\mathrm{C}_{8} \mathrm{H}_{9} \mathrm{NO}_{3}$ & Others & -2.295 & 0.0031 & 1.207 \\
\hline
\end{tabular}

${ }^{*}$ Only variance for component 1 has been shown. 
the delta value of 1.6, FDR of 0.001 and with less than one (0.5) false positive. Similarly the most important metabolites were also identified by PLS-DA method based on the VIP score using five-component model. Random Forest classification ranked the important metabolites in order of decreasing prediction accuracy (Mean Decrease Accuracy) using 5000 trees (permutation) with an overall (OOB, out-of-bag) error of 0.0417. Overall the results were quite similar across all three methods. The top most important 60 metabolites which were identified by at least 2 different methods are shown in Table 2. These important metabolites included different amino acids, sugars, organic acids/compounds, amines, fatty acids and others.

Over the course of 24 hours 89 metabolites exhibited significant fluctuations (ANOVA, $\mathrm{P} \leq 0.05$, Fisher's LSD) across the 8 sampled time-points between the two treatments (Supplemental Table S2). The most significant metabolites including tryptophan, proline, pipecolate, linamarin, indoline and phenylalanine remained significantly higher under drought stress compared to control across all 8 time points with the highest peak at $18 \mathrm{~h}(3 \mathrm{pm})$ (Figure 3$)$. On the contrary, there were several sugars, fatty acid, organic acids, polyamines and amino acids which were significantly higher in control compared to drought stress across most or all of time points (Figure 3). Fluctuations were also seen based on day and night cycles. For example, levels of serine, aspartate, raffinose, myo-inositol, spermidine, gluconic acid, DIBOA-glucoside and tribenuron methyl increased beginning at $06 \mathrm{~h}$ and remained high during the day time and then decreased at night $(21 \mathrm{~h})$ in control leaves, whereas their levels were low during day time but increased at or after $24 \mathrm{~h}$ during night in drought stressed leaves.

To better elucidate the biological functions of identified metabolites, a pathway analysis was performed using Oryzasativa and Arabidopsis thaliana as the pathway libraries. As expected, these metabolites were involved in number of different pathways (Table 3). As the rice and Arabidopsis pathway libraries do not have all the compounds, KEGG website was also used to find their metabolic pathways.

\subsection{Identification of Metabolite Biomarkers}

Applying a ROC (Receiver Operating Characteristic)-curve based approach of biomarker analysis [33], MetaboAnalyst 3.0 identified eight metabolites with high AUC values (Area under ROC curve, $>0.84$ ) as potential biomarkers for drought tolerance based on classical univariate ROC curve analysis module. These included $\mathrm{N}$-acetyl-glutamic acid, L-tryptophan, L-proline, linamarin, pipecolate, L-isoleucine, malonate and piperidine (Figure 4). The highest AUC value (0.92) with highest sensitivity (0.9) and specificity (0.9) was found for $\mathrm{N}$-acetyl-glutamic acid followed by tryptophan (AUC: $0.90 ; 0.9,0.8$ ) and proline (AUC: $0.89,1,0.9$ ). Out of these 8 potential biomarkers, 6 metabolites were also predicted as potential biomarkers by multivariate ROC curve exploration module, 


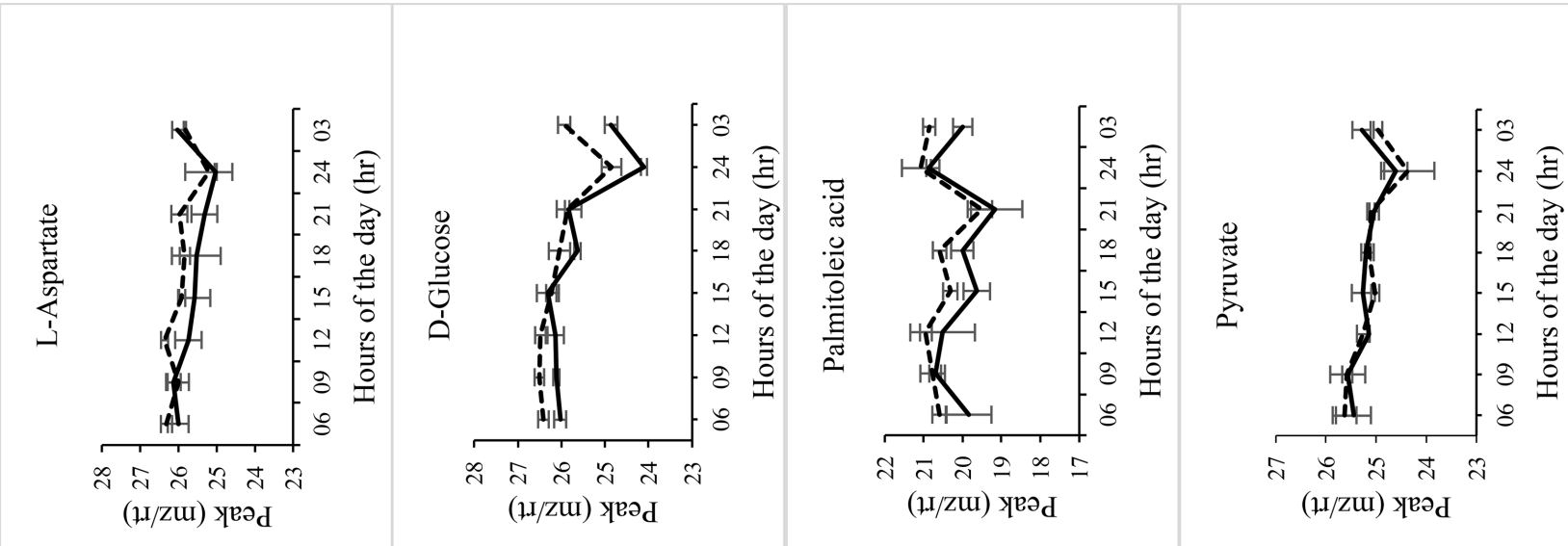

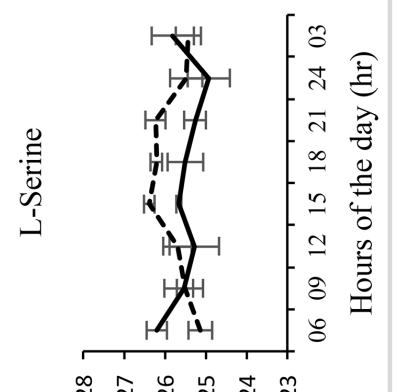

$\stackrel{\sim}{\sim} \stackrel{n}{\sim} \cong$

(1. /ZU) Yएव,

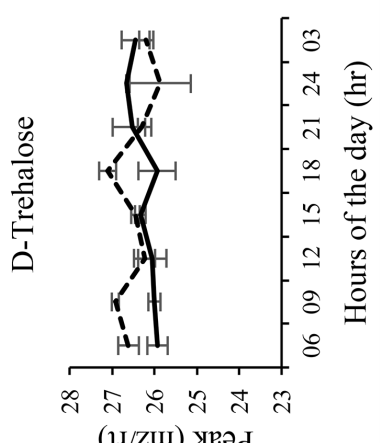

(1//ZU) урәд
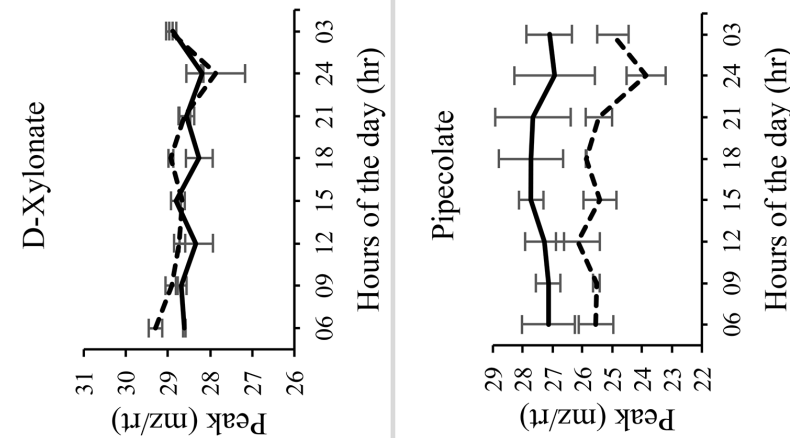

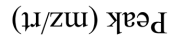

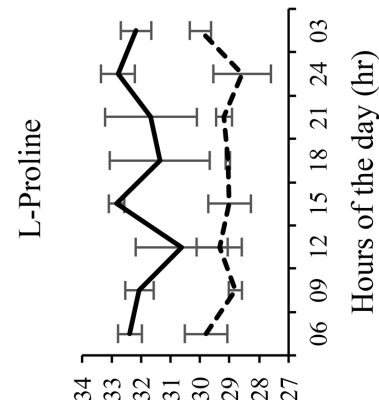

(1I/ZU) yएə⿸厂

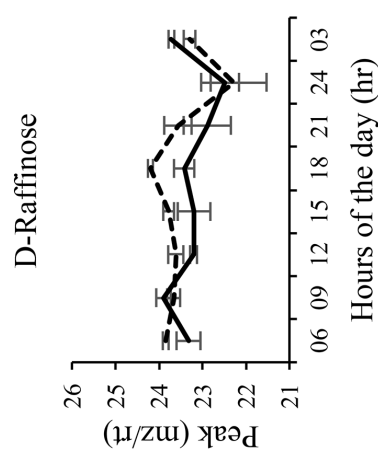

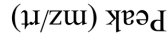
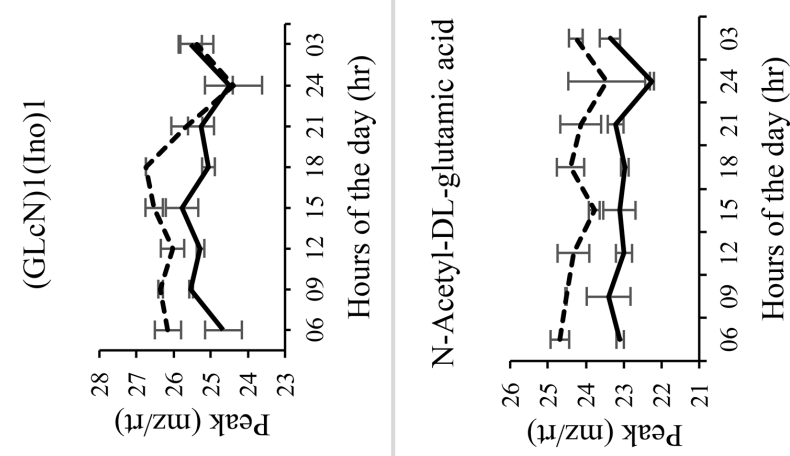
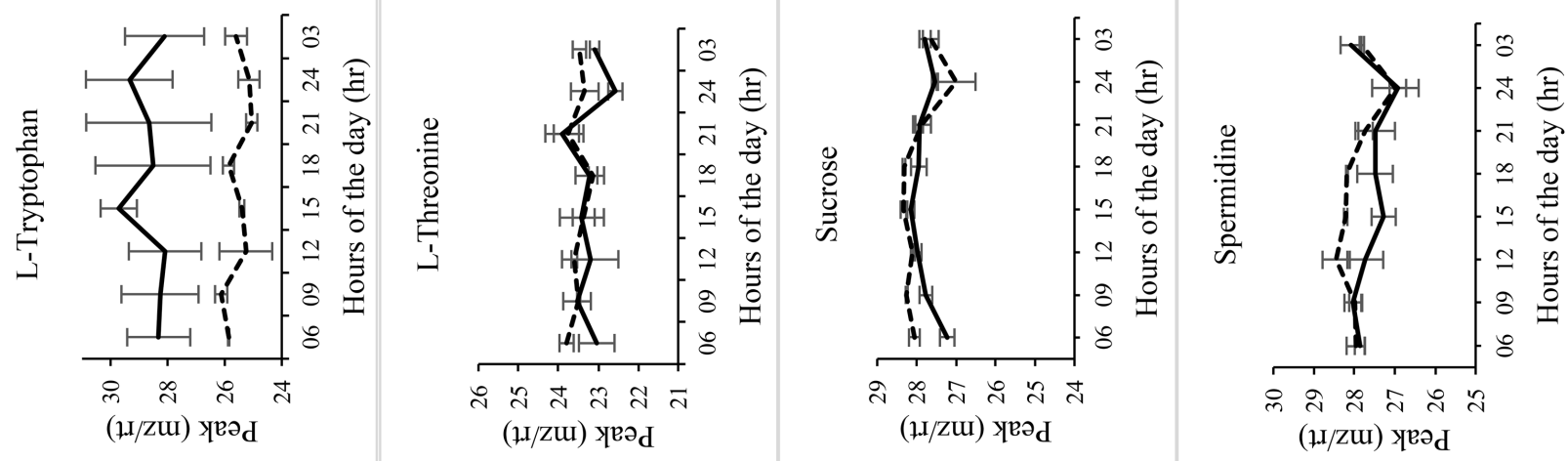

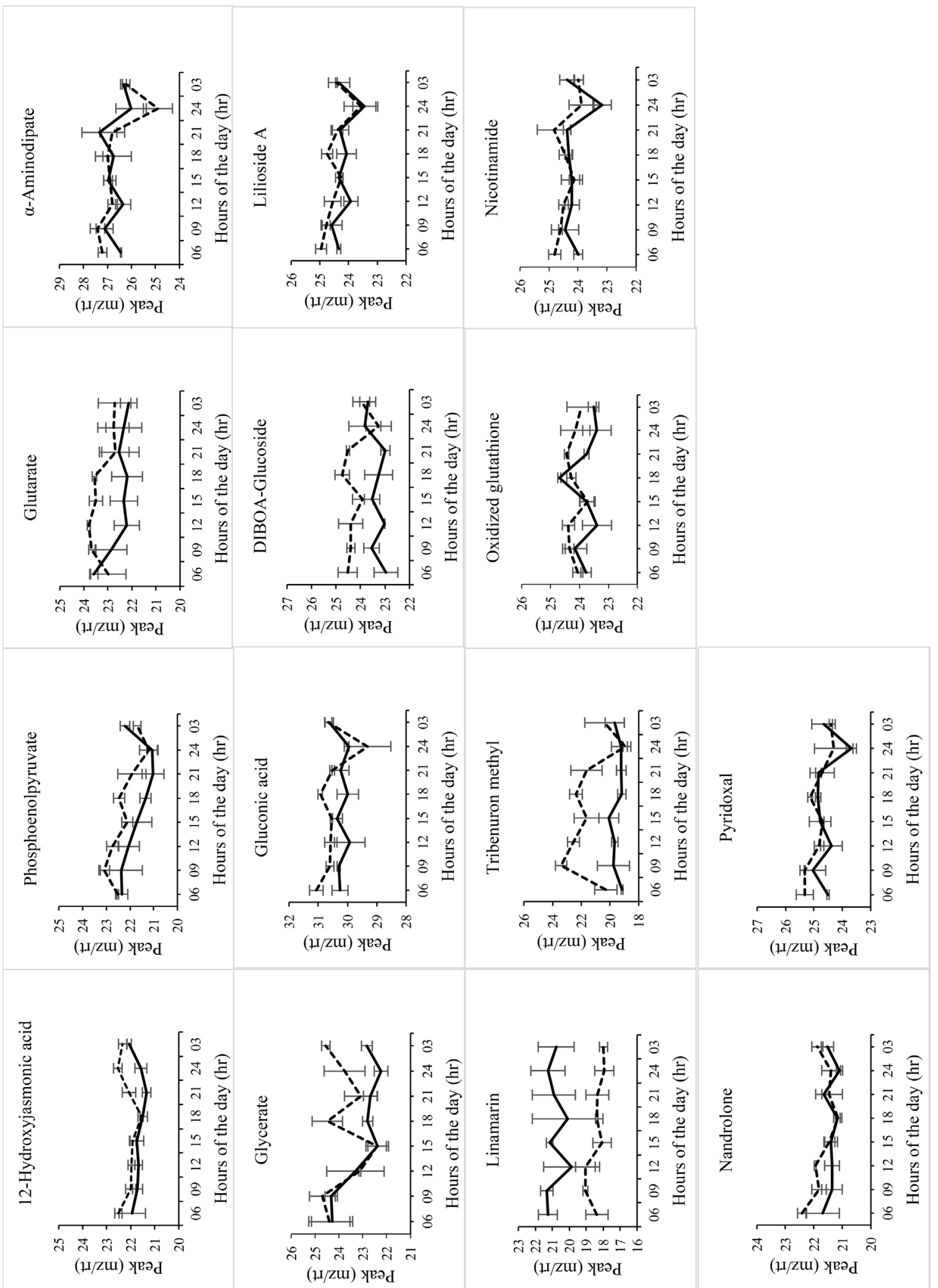

Figure 3. Significantly different levels of selected metabolites (ANOVA, P $\leq 0.05$, Fisher's LSD) in the wheat flag leaves at 8 different time points $(03 \mathrm{~h}, 06 \mathrm{~h}, 09 \mathrm{~h}, 12 \mathrm{~h}, 15 \mathrm{~h}, 18 \mathrm{~h}, 21 \mathrm{~h}, 24 \mathrm{~h})$ during 24 hours of day-night period under drought and control conditions. Dotted line (----) and solid line indicate the metabolite levels under control and drought conditions, respectively. Error bars represent standard errors of the mean at each time point. 
Table 3. Selected metabolites and their involvement in different pathways identified by Pathway Analysis of MetaboAnalist 3 using Oryzasativa japonica and Arabidopsis thaliana as the pathway libraries.

\begin{tabular}{|c|c|}
\hline Compound & Pathway involved \\
\hline L-Proline & Arginine and proline metabolism, Aminoacyl-tRNA biosynthesis \\
\hline L-Tryptophan & $\begin{array}{l}\text { Glycine, serine and threonine metabolism, Aminoacyl-tRNA biosynthesis, Phenylalanine, tyrosine and } \\
\text { tryptophan biosynthesis (Shikimate pathway), Glucosinolate biosynthesis, Tryptophan metabolism, }\end{array}$ \\
\hline 4-Aminobutanoate (GABA) & Arginine and proline metabolism, Alanine, aspartate and glutamate metabolism, Butanoate metabolism \\
\hline L-Serine & $\begin{array}{l}\text { Glycine, serine and threonine metabolism, Aminoacyl-tRNA biosynthesis, Cyanoamino acid metabolism, } \\
\text { Cysteine and methionine metabolism, Methane metabolism, Sulfur metabolism, Sphingolipid metabolism }\end{array}$ \\
\hline Threonine & Aminoacyl-tRNA biosynthesis, Valine, leucine and isoleucine biosynthesis \\
\hline Sucrose & Galactose metabolism, Starch and sucrose metabolism \\
\hline Raffinose & Galactose metabolism \\
\hline N-Acetyl-DL-glutamic acid & Arginine and proline metabolism \\
\hline Aspartate & $\begin{array}{l}\text { Glycine, serine and threonine metabolism, Arginine and proline metabolism, Carbon fixation in photosynthetic } \\
\text { organisms, Alanine, aspartate and glutamate metabolism, Aminoacyl-tRNA biosynthesis, Cyanoamino acid } \\
\text { metabolism, beta-Alanine metabolism, Cysteine and methionine metabolism, Lysine biosynthesis, Nicotinate } \\
\text { and nicotinamide metabolism }\end{array}$ \\
\hline Pyruvate & $\begin{array}{l}\text { Glycine, serine and threonine metabolism, Carbon fixation in photosynthetic organisms, Alanine, aspartate and } \\
\text { glutamate metabolism, Cysteine and methionine metabolism, Citrate cycle (TCA cycle), Butanoate metabolism, } \\
\text { Pyruvate metabolism, C5-Branched dibasic acid metabolism, Glycolysis or Gluconeogenesis, Valine, leucine } \\
\text { and isoleucine biosynthesis, Pantothenate and CoA biosynthesis, Terpenoid backbone biosynthesis }\end{array}$ \\
\hline Phosphoenolpyruvic acid & $\begin{array}{l}\text { Carbon fixation in photosynthetic organisms, Citrate cycle (TCA cycle), Pyruvate metabolism, Phenylalanine, } \\
\text { tyrosine and tryptophan biosynthesis, Glycolysis or Gluconeogenesis }\end{array}$ \\
\hline Nicotinate & Nicotinate and nicotinamide metabolism \\
\hline Glyceric acid & Glycerolipid metabolism \\
\hline Oxidized glutathione & Glutathione metabolism \\
\hline Spermidine & Arginine and proline metabolism, beta-Alanine metabolism, Glutathione metabolism \\
\hline Palmitoleic acid & Fatty acid biosynthesis \\
\hline Pyridoxal & Vitamin B6 metabolism \\
\hline Linamarin & Cyanoamino acid metabolism; biosynthesis of secondary metabolites \\
\hline Pipecolate & Lysine degradation; biosynthesis of secondary metabolites \\
\hline Malonate & Pyrimidine metabolism; beta-Alanine metabolism \\
\hline Alpha-Aminoadipate & Lysine biosynthesis; lysine degradation; biosynthesis of secondary metabolites \\
\hline Piperidine & Tropane, piperidine and pyridine alkaloid biosynthesis; protein digestion and absorption \\
\hline
\end{tabular}



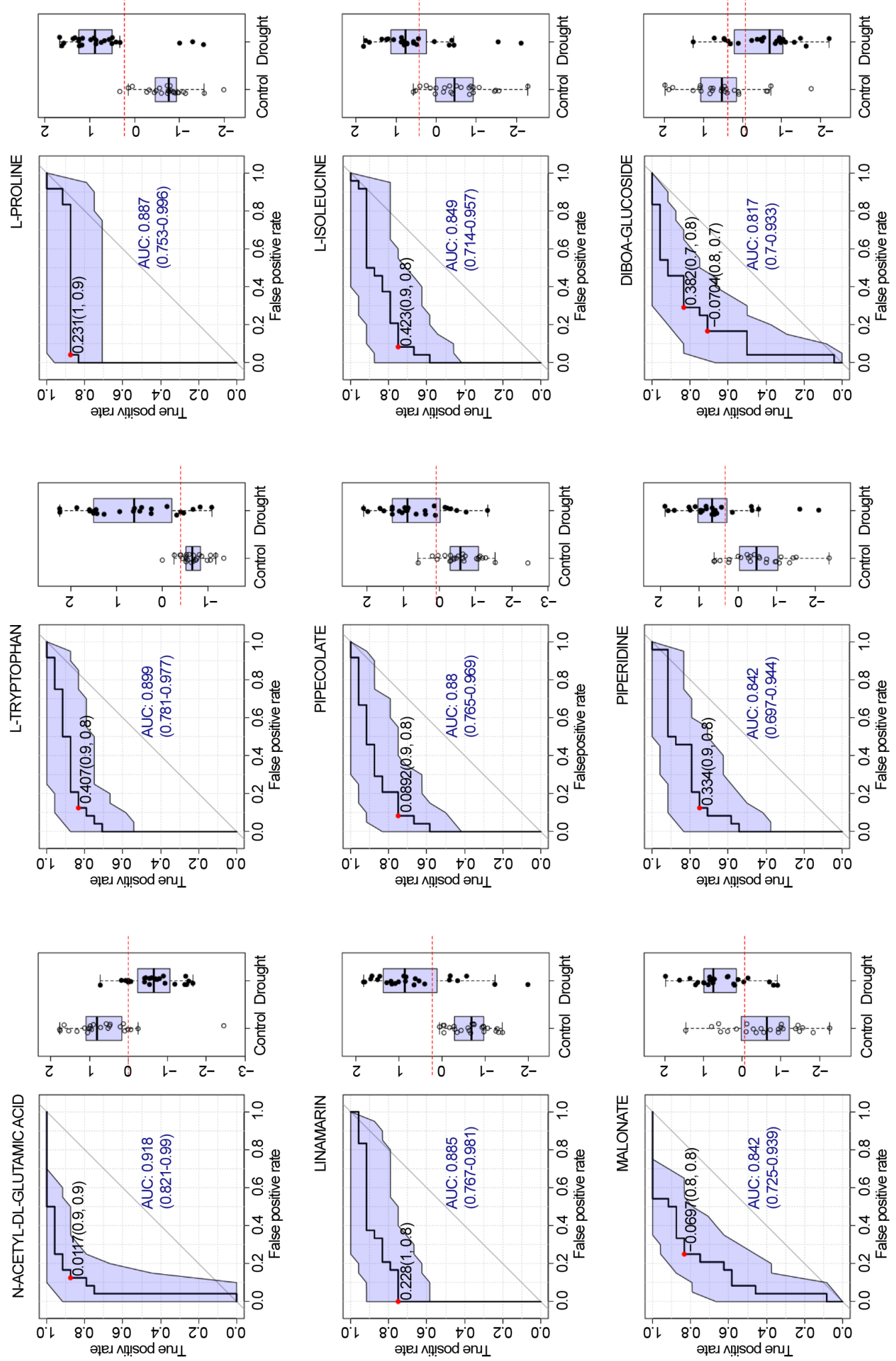

Figure 4. Potential biomarkers predicted by classical univariate ROC curve analysis. The left-side plot of the individual metabolite figure is showing ROC (receiver operating characteristics) curve with 95\% confidence interval (shadowed) and the solid red dot indicates the optimal cutoff with the associated sensitivity and specificity values. The right-side box-and-whisker plot shows the distribution of abundance values of individual metabolite in control and drought sample with the optimal cutoff as a horizontal dotted red line. 
namely, $\mathrm{N}$-acetyl-glutamic acid, L-proline, L-tryptophan, linamarin, pipecolate, and malonate (Figure 5). In multivariate module, biomarker (feature) selection, ROC curve-based model building and performance evaluation are performed multiple times using Monte-Carlo cross validation (MCCV). The potential biomarkers, L-proline, DIBOA-glucoside $\mathrm{N}$-acetyl-glutamic acid, malonate, pipecolate and tryptophan have the selection frequency of 1.0 (i.e. selected $100 \%$ of the time in the model) on the SVM feature selection algorithm. The metabolite DIBOA-glucoside having selection frequency of 1.0 in multivariate module also
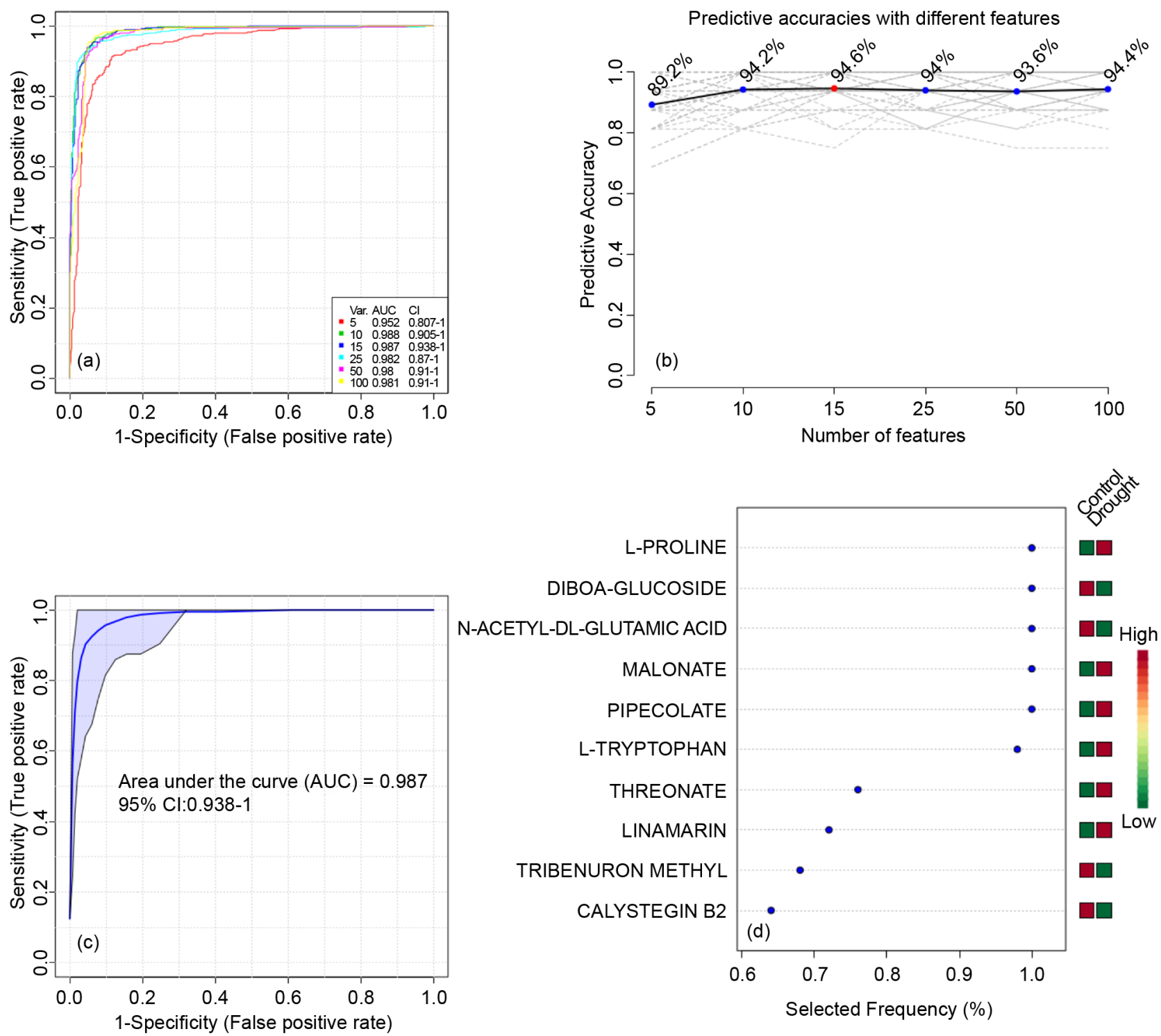

Figure 5. Biomarker identification/prediction by Multivariate ROC curve based exploratory analysis. The ROC curves were generated using an algorithm based on Monte-Carlo cross validation (MCCV) through balanced subsampling coupled with linear SVM (Support vector machine) for the classification method and SVM built-in for the feature ranking method. (a) Overview of all ROC curves created by MetaboAnalyst from 6 different biomarker models using different number of features (5, 10, 15, 25, 50 and 100) with their corresponding AUC value and confidence interval; (b) Graph showing the predictive accuracies of 6 different biomarker models. The red dot shows the highest accuracy for the 15-feature panel of model 3; (c) ROC curve for selected biomarker model 3; (d) Top 10 potential biomarkers predicted based on their frequencies of being selected during cross validation. 
had high AUC value of 0.82 and high sensitivity (0.8) and specificity (0.7) in univariate analysis.

\section{Discussion}

Drought stress can severely affect physiological and morphological mechanisms that could potentially affect performance, functionality and ultimately survival of plants. To maintain growth and productivity, plants must adapt to stress conditions by exercising specific tolerance mechanisms. The alternation of different attributes related to photosynthesis is a good indicator of stress tolerance. Damage to photosynthetic capacity due to water stress during grain filling can limit supply of assimilates to growing grain and thus reduces yield potential. Chlorophyll fluorescence, SPAD chlorophyll content, and electrolyte leakage or plasma membrane damage are very sensitive and quick responding variable for monitoring water stress. In the present study, drought stressed flag leaves exhibited a reduction of chlorophyll fluorescence, SPAD chlorophyll content and higher electrolyte leakage, which indicate significant damage to photosystem II and the plasma membrane due to drought stress at grain filling stage of wheat [34] [35]. This declining photochemical activity and membrane integrity of wheat plants (SS8641) under drought was also reflected in lower shoot dry biomass, grains spike $^{-1}$, grain weight plant ${ }^{-1}$ and harvest index compared to the control.

In addition to physiological and morphological traits, levels of metabolite accumulation during stress at a particular growth stage can provide a more specific and accurate indication of stress tolerance. In the present study, the non-targeted metabolite profiling revealed significant changes in 152 metabolites in wheat flag leaves under drought stress condition compared to control. Of these differentially accumulated metabolites, 60 metabolites were identified as the most important metabolites by three different methods: PLS-DA, RF and SAM, which included an array of different amino acids, sugars, organic acids or compounds, polyamines, fatty acids and their derivatives. Amino acids including tryptophan, proline, phenylalanine, tyrosine and isoleucine accumulated up to 7.1 fold in drought treated flag leaves compared to control, although the levels of some amino acids (e.g. serine, glutathione, glutamine, GABA, threonine) decreased due to drought stress. The accumulation of amino acids under drought stress has been reported previously in several studies [18] [21] [36]. An increased level of amino acids is considered to improve stress tolerance in plants through different mechanisms, such as osmotic adjustment, detoxification of reactive oxygen species, and intracellular $\mathrm{pH}$ regulation (see review by Krasensky and Jonak 2012 [37]).

Tryptophan acts as an osmolyte in ion transport regulation and in modulating stomatal opening, and maintaining water balance between air and plant [38]. Increased accumulation of tryptophan in wheat under drought is also supported by previous studies [21] [39] [40] [41]. Higher levels of tyrosine and phenylalanine under drought stress are also consistent with a study by Bowne et al. (2012) 
[21]. The aromatic amino acids, tryptophan, tyrosine and phenylalanine are the precursors of different secondary metabolites including indoleacetate, lipid precursor and lignin in shikimate pathway, which plays a vital role in stress tolerance [42] [43] [44].

Proline level was increased in flag leaves under drought conditions and the accumulation of proline has been correlated with stress tolerance in a wide range of plants by different scientists [45] [46]. Proline serves as a compatible solute and helps plants to avoid oxidative stress by preserving osmotic balance or cell turgor, stabilizing membranes, stopping electrolyte leakage, and by keeping reduced concentrations of reactive oxygen species (ROS) [47] [48] [49]. Furthermore, the increased level of branched chain amino acids (e.g. isoleucine) under drought stress was reported in tolerant cultivars of wheat [21] [39] [40], barley [50], and Arabidopsis [51], and similar result was found in this study. Taylor et al. (2004) reported that branched chain amino acids are a source of alternate energy when Arabidopsis plants were under sugar starvation, which is a common phenomenon under stress [52].

Sugars and their derivatives were significantly reduced under drought stress. Lower accumulation of glucose and sucrose is potentially due to reduced photosynthetic capacity, as demonstrated by lower chlorophyll fluorescence and SPAD chlorophyll content, of the drought stressed leaves at the grain filling stage [34] [35]. In addition, enhanced maturity under stress condition may have resulted in increased partitioning of carbohydrates from source (leaf) to sink (grain). Depleted starch content in drought-stressed barley flag leaves compared to control has been reported under stress condition [53]. Trehalose is reported to be accumulated at a moderate level under abiotic stress and serves as an osmolyte to stabilize proteins and membranes [54] [55]. The trehalose of drought stressed leaves in our study may have been utilized as an osmolyte to provide the drought tolerance observed in the genotype SS8641. Myo-inositol acts as precursor of many metabolites especially raffinose that are involved in stress tolerance by providing membrane stability and antioxidative functions [56]. Decreased levels of raffinose of the drought stressed leaves can be accounted to the drought tolerance of the wheat plants (SS8641). Obata et al. (2015) also reported lower levels of raffinose and myo-inositol in a tolerant maize genotype and suggested that that these could be due to the use of raffinose family oligosacchardises (RFOs) by the tolerant genotypes as their carbon sources [15].

The lower accumulation of sugars was further accompanied by reduced levels of organic acids or compounds including pyruvate, phosphoenolpruvate, a-ketoglutaric acid which are involved in Krebs cycle in leaves of stressed plants. Drought stress in our study didn't trigger higher accumulation of organic acids or compounds except pipecolate and linamarin. A decreased level of organic acids or compounds was reported in wheat [21] and soybean [36] under drought stress in tolerant genotypes. In contrast, the consistent higher accumulation of pipecolate and linamarin at all time points upon drought stress indicates their 
potential role in drought tolerance of wheat.

Pipecolate is reported to have a defensive action on proteins and nucleic acid structures by maintaining a stable osmotic status in plants under variable soil water and salt stress [57]. Bowne et al. (2012) also found significantly changed levels of pipecolate among wheat leaves grown under drought stress compared to control [21]. Drought stress led to a significantly higher accumulation of linamarin level in the leaves. Linamarin is a cyanogenic glycoside, which plays an important role in nitrogen transport [58] and can act as an immediate chemical defense against insect and pathogen infestation by discharging toxic cyanide [59]. Higher level of linamarin was reported in cassava leaf under drought stress [14]. Our study first demonstrated a consistent higher accumulation of linamarin under drought stress at different time points in wheat flag leaves which indicates that linamarin has a strong relationship with drought tolerance in wheat at grain filling stage. The accumulated linamarin under drought stress could further be hydrolysed to release hydrogen cyanide, which can act as precursor of amino acids. This is potentially attributed to higher accumulation of amino acids under drought conditions in our study.

The level of different metabolites changed in wheat flag leaves at 8 different time points across a diel cycle and they changed differently under drought and control conditions (Figure 3, Supplemental Table S2). Metabolites showed mostly 3 different diel patterns of accumulation in flag leaves at 8 different time points. Most of the amino acids (e.g. tryptophan, proline, indoline, phenylalanine) and some organic acids (e.g. pipecolate, linamarin) were accumulated and remained higher in drought stressed leaves over the $24 \mathrm{hrs}$ of day-night period with the maximum accumulation at $3 \mathrm{pm}$ compared to controls. But the levels of sugars, fatty acid, organic acids, amines and some amino acids were lower in drought plants over the most or all of time points compared to controls. Our study also showed that the levels of serine, aspartate, raffinose, myo-inositol, spermidine, gluconic acid, DIBOA-glucoside and tribenuron methyl remained higher during day time but decreased at night under control condition but the diel pattern of metabolite accumulation became completely opposite under drought condition. This pattern of metabolite accumulation was also reported by Benard et al. (2015) [60] where sucrose and serine were accumulated during day time and decreased at night under control condition.

The pathway analysis by using MetaboAnalyst was able to link with 33 metabolic and biosynthesis pathways of the KEGG pathway database and Rice Annotation Project database (Table 3 ). The aromatic amino acids such as phenylalanine, tyrosine and tryptophan are produced via the shikimate pathway having fundamental roles in plant reproduction, development, pest-defense, and environmental stresses [43] [61]. Serine, glycine, and threonine all can be converted to the 3-carbon a-ketoacid pyruvate or pyruvate which was downregulated in this study under drought stress. Pyruvate is the end product of glycolysis in the cytosol, and could be transported by mitochondrial pyruvate carriers (MPCs) 
into mitochondria for consequent cellular substance and energy metabolism [62]. Arginine and proline metabolism is one of the unique pathways for the biosynthesis of the amino acids. The regulatory mechanisms controlling proline metabolism, intercellular and intracellular transport, and connections of proline to other metabolic pathways are all imperative to the in vivo functions of proline metabolism [63]. Proline accumulated mostly in the cytosol due to water stress reduces cytoplasmic acidosis and conserves $\mathrm{NADP}+/ \mathrm{NADPH}$ ratios well-suited with metabolism [64]. Upon relief of stress, the proline metabolic pathway reduces mitochondrial oxidative phosphorylation and produces ATP for repairing of stress-induced damages [49] [64]. The increased or decreased levels of metabolites in our study integrated with their linked major metabolic pathways and biosynthetic processes presents a complicated process of drought stress responses in wheat.

We also conducted the analysis to find potential metabolite biomarkers associated with drought tolerance in wheat for the screening of drought tolerant genetic resources of wheat. Obata et al. (2015) opined that promising metabolic traits were stronger in explaining variability in maize grain yield than classical agronomic yield components [15]. Six metabolites namely $\mathrm{N}$-acetyl-glutamic acid, tryptophan, proline, linamarin, pipecolate, malonate and DIBOA-glucoside were identified as potential biomarkers by classical univariate and multivariate analysis. The finding of proline and tryptophan accumulation in drought tolerant wheat genotype was not something new. They were already reported as associated with their abiotic stress tolerance by several studies in different crops [37]. But the identification of $\mathrm{N}$-acetyl-glutamic acid, linamarin, pipecolate and DIBOA-glucoside in wheat genotype to be associated with drought tolerance was not reported earlier and could be promising metabolite biomarkers for screening the tolerant genotypes. However, further investigation needs to be conducted in a panel of wheat germplasm with different genetics backgrounds to draw a comprehensive conclusion.

\section{Conclusion}

This study is the first study in wheat which used a non-targeted LC-HRMS Isotopic Ratio Outlier Analysis (IROA) Global Metabolomics method to identify accumulated metabolites in flag leaves under drought and control conditions at 8 time points during 24 hours day-night period. The study reports increased or decreased level of numerous metabolites simultaneously and consistently at different time points. Some of metabolites received considerable research attention under drought stress, but many of these did not. Metabolites like tryptophan, proline, pipecolate, linamarin, $\mathrm{N}$-acetyl-glutamic acid, DIBOA-glucoside accrued to a greater level after drought stress, which was likely the indication of acclimation in responses to the drought stress. Elevated tryptophan and proline are common in other crops and are not surprising, but increased levels of pipcolate, linamarin, and decreased levels of $\mathrm{N}$-acetyl-glutamic acid and DIBOA-glucoside 
in response to drought stress have not been widely reported. These metabolites may serve as metabolite biomarkers for screening drought stress tolerance in wheat germplasms to develop climate resilient wheat. The finding of these metabolites could potentially be the effect of genetic variation, as we used soft wheat which is different than other wheat genotypes used in the study before, including in Bowne et al. (2012) [21]. This difference indicates that global wheat community needs to use diverse set of germplasms for studying metabolite accumulation under drought stress. That will improve our understanding on metabolic control of complex stress conditions including drought in wheat. In addition, we employed LC-HRMS analysis with IROA method which was not also reported in wheat before. This technique is sensitive and potentially more robust than Nuclear magnetic resonance (NMR) and gas chromatography-mass spectrometry (GC-MS) technology as it can identify metabolites in low concentration and has better capacity to control FDR rate. Although some of these newly identified metabolites are promising as biomarkers for improving drought stress tolerance in wheat, their correlation to drought tolerance in wheat and other cereal crops requires further investigation.

\section{Acknowledgements}

We are grateful to Southeast Center for Integrated Metabolomics (SECIM), University of Florida for all valuable assistance for metabolic profiling and data cleaning for further analysis.

\section{Funding}

This work was supported by the Dean's Research Initiative, IFAS, University of Florida.

\section{Conflict of Interest}

The authors declare that they have no conflict of interests.

\section{References}

[1] Weigand, C. (2011) Wheat Import Projections Towards 2050. US Wheat Associates, USA.

[2] Dai, A. (2013) Increasing Drought under Global Warming in Observations and Models. Nature Climate Change, 3, 171. https://doi.org/10.1038/nclimate1811

[3] Lobell, D.B., Roberts, M.J., Schlenker, W., Braun, N., Little, B.B., Rejesus, R.M. and Hammer, G.L. (2014) Greater Sensitivity to Drought Accompanies Maize Yield Increase in the U.S. Midwest. Science, 344, 516-519. https://doi.org/10.1126/science.1251423

[4] Ruane, A.C., McDermid, S., Rosenzweig, C., Baigorria, G.A., Jones, J.W., Romero, C.C. and DeWayne, C.L. (2014) Carbon-Temperature-Water Change Analysis for Peanut Production under Climate Change: A Prototype for the AgMIP Coordinated Climate-Crop Modeling Project (C3MP). Global Change Biology, 20, 394-407. https://doi.org/10.1111/gcb.12412 
[5] Wang, W., Vinocur, B. and Altman, A. (2003) Plant Responses to Drought, Salinity and Extreme Temperatures: Towards Genetic Engineering for Stress Tolerance. Planta, 218, 1-14. https://doi.org/10.1007/s00425-003-1105-5

[6] Richards, R.A., Condon, A.G. and Rebetzke, G.J. (2001) Traits to Improve Yield in Dry Environments. In: Reynolds, M.P., Ortiz-Monasterio, J.I. and McNab, A., Eds., Application of Physiology in Wheat Breeding, CIMMYT, Mexico, 88-100.

[7] Yang, J., Zhang, J., Huang, Z., Zhu, Q. and Wang, L. (2000) Remobilization of Carbon Reserves Is Improved by Controlled Soil-Drying during Grain Filling of Wheat. Crop Sciences, 40, 1645-1655. https://doi.org/10.2135/cropsci2000.4061645x

[8] Yang, J., Zhang, J., Wang, Z., Zhu, Q. and Liu, L. (2001) Water Deficit-Induced Senescence and Its Relationship to the Remobilization of Pre-Stored Carbon in Wheat during Grain Filling. Agronomy Journal, 93, 196.

https://doi.org/10.2134/agronj2001.931196x

[9] Zhang, J., Sui, X., Li, B., Su, B., Li, J. and Zhou, D. (1998) An Improved Water-Use Efficiency for Winter Wheat Grown under Reduced Irrigation. Field Crops Research, 59, 91-98. https://doi.org/10.1016/S0378-4290(98)00104-X

[10] Kobata, A.T., Palta, J. and Turner, N.C. (1992) Rate of Development of Postanthesis Water Deficits and Grain Filling of Spring Wheat. Crop Sciences, 32, 1238. https://doi.org/10.2135/cropsci1992.0011183X003200050035x

[11] Schilmiller, A.L., Pichersky, E. and Last, R.L. (2012) Taming the Hydra of Specialized Metabolism: How Systems Biology and Comparative Approaches Are Revolutionizing Plant Biochemistry. Current Opinion in Plant Biology, 15, 338-344. https://doi.org/10.1016/j.pbi.2011.12.005

[12] Kim, S.G., Yon, F., Gaquerel, E., Gulati, J. and Baldwin, I.T. (2011) Tissue Specific Diurnal Rhythms of Metabolites and Their Regulation during Herbivore Attack in a Native Tobacco, Nicotiana attenuata. PLoS ONE, 6, e26214. https://doi.org/10.1371/journal.pone.0026214

[13] Shuib, N.H., Shaari, K., Khatib, A., Kneer, R., Zareen, S., Raof, S.M., Lajis, N.H. and Neto, V. (2011) Discrimination of Young and Mature Leaves of Melicope ptelefolia Using 1H NMR and Multivariate Data Analysis. Food Chemistry, 126, 640-645. https://doi.org/10.1016/j.foodchem.2010.10.043

[14] Zhao, P., Liu, P., Shao, J., Li, C., Wang, B., Guo, X., Yan, B., Xia, Y. and Peng, M. (2015) Analysis of Different Strategies Adapted by Two Cassava Cultivars in Response to Drought Stress: Ensuring Survival or Continuing Growth. Journal of $E_{X-}$ perimental Botany, 66, 1477-1488. https://doi.org/10.1093/jxb/eru507

[15] Obata, T., Witt, S., Lisec, J., Palacios-Rojas, N., Florez-Sarasa, I., Yousfi, S., Araus, J.L., Cairns, J.E. and Fernie, A.R. (2015) Metabolite Profiles of Maize Leaves in Drought, Heat and Combined Stress Field Trials Reveal the Relationship between Metabolism and Grain Yield. Plant Physiology, 169, 2665-2683.

[16] Khakimov, B., Jespersen, B. and Engelsen, S. (2014) Comprehensive and Comparative Metabolomic Profiling of wheat, Barley, Oat and Rye Using Gas Chromatography-Mass Spectrometry and Advanced Chemometrics. Foods, 3, 569-585. https://doi.org/10.3390/foods3040569

[17] Zhao, X., Wang, W., Zhang, F., Deng, J., Li, Z. and Fu, B. (2014) Comparative Metabolite Profiling of Two Rice Genotypes with Contrasting Salt Stress Tolerance at the Seedling Stage. PLoS ONE, 9, e108020. https://doi.org/10.1371/journal.pone.0108020

[18] Hochberg, U., Degu, A., Toubiana, D., Gendler, T., Nikoloski, Z., Rachmilevitch, S. and Fait, A. (2013) Metabolite Profiling and Network Analysis Reveal Coordinated 
Changes in Grapevine Water Stress Response. BMC Plant Biology, 13, 184. https://doi.org/10.1186/1471-2229-13-184

[19] Chakraborty, U. and Pradhan, B. (2012) Drought Stress-Induced Oxidative Stress and Antioxidative Responses in Four Wheat (Triticum aestivum L.) Varieties. Archives of Agronomy and Soil Science, 58, 617-630. https://doi.org/10.1080/03650340.2010.533660

[20] Hura, T., Hura, K., Dziurka, K., Ostrowska, A., Bączek-Kwinta, R. and Grzesiak, M. (2012) An Increase in the Content of Cell Wall-Bound Phenolics Correlates with the Productivity of Triticale under Soil Drought. Journal of Plant Physiology, 169, 1728-1736. https://doi.org/10.1016/j.jplph.2012.07.012

[21] Bowne, J.B., Erwin, T.A., Juttner, J., Schnurbusch, T., Langridge, P., Bacic, A. and Roessner, U. (2012) Drought Responses of Leaf Tissues from Wheat Cultivars of Differing Drought Tolerance at the Metabolite Level. Molecular Plant, 5, 418-429. https://doi.org/10.1093/mp/ssr114

[22] Sumner, L.W., Mendes, P. and Dixon, R.A. (2003) Plant Metabolomics: Large-Scale Phytochemistry in the Functional Genomics Era. Phytochemistry, 62, 817-836. https://doi.org/10.1016/S0031-9422(02)00708-2

[23] Fernie, A.R. and Schauer, N. (2009) Metabolomics-Assisted Breeding: A Viable Option for Crop Improvement? Trends in Genetics, 25, 39-48.

https://doi.org/10.1016/j.tig.2008.10.010

[24] Stupp, G.S., Clendinen, C.S., Ajredini, R., Szewc, M.A., Garrett, T., Menger, R.F., Yost, R.A., Beecher, C. and Edison, A.S. (2013) Isotopic Ratio Outlier Analysis Global Metabolomics of Caenorhabditis elegans. Analytical Chemistry, 85, 11858-11865. https://doi.org/10.1021/ac4025413

[25] Borrill, P., Fahy, B., Smith, A.M. and Uauy, C. (2015) Wheat Grain Filling Is Limited by Grain Filling Capacity Rather than the Duration of Flag Leaf Photosynthesis: A Case Study Using NAMRNAi Plants. PLOS ONE, 10, e0134947. https://doi.org/10.1371/journal.pone.0134947

[26] Sanchez-Bragado, R., Elazab, A., Zhou, B., Serret, M.D., Bort, J., Nieto-Taladriz, M.T. and Araus, J.L. (2014) Contribution of the Ear and the Flag Leaf to Grain Filling in Durum Wheat Inferred from the Carbon Isotope Signature: Genotypic and Growing Conditions Effects. Journal of Integrative Plant Biology, 56, 444-454. https://doi.org/10.1111/jipb.12106

[27] Kadir, S., Von Weihe, M. and Al-Khatib, K. (2007) Photochemical Efficiency and Recovery of Photosystem II in Grapes after Exposure to Sudden and Gradual Heat-Stress. Journal of the American Society for Horticultural Science, 132, 764-769.

[28] Ristic, Z., Bukovnik, U. and Prasad, P.V.V. (2007) Correlation between Heat Stability of Thylakoid Membranes and Loss of Chlorophyll in Winter Wheat under Heat Stress. Crop Science, 47, 2067. https://doi.org/10.2135/cropsci2006.10.0674

[29] Talukder, S.K., Babar, M.A., Vijayalakshmi, K., Poland, J., Prasad, P.V., Bowden, R. and Fritz, A. (2014) Mapping QTL for the Traits Associated with Heat Tolerance in Wheat (Triticum aestivum L.). BMC Genetics, 15, 97. https://doi.org/10.1186/s12863-014-0097-4

[30] Ristic, Z. and Cass, D.D. (1993) Dehydration Avoidance and Damage to the Plasma and Thylakoid Membranes in Lines of Maize Differing in Endogenous Levels of Abscisic Acid. Journal of Plant Physiology, 142, 759-764. https://doi.org/10.1016/S0176-1617(11)80915-6

[31] Dong, F., Beecher, C., Akond, M., Erickson, J. and Babar, M.A. (2015) Metabolic 
Effect of Drought Stress during the Grain Filling Growth Stage in Wheat Measured by Isotopic Ratio Outlier Analysis (IROA). 63rd ASMS Conference on Mass Spectrometry, 31May-4 June 2015, America's Center, St. Louis, Missouri, USA.

[32] Qiu, Y., Moir, R., Willis, I., Beecher, C., Tsai, Y., Garrett, T., Yost, R. and Kurland, J. (2016) Isotopic Ratio Outlier Analysis of the S. cerevisiae Metabolome Using Accurate Mass Gas Chromatography/Time-of-Flight Mass Spectrometry: A New Method for Discovery. Analytical Chemistry, 88, 2747-2754.

https://doi.org/10.1021/acs.analchem.5b04263

[33] Xia, J. and Wishart, D.S. (2016) Using Metabo Analyst 3.0 for Comprehensive Metabolomics Data Analysis. Current Protocols in Bioinformatics, 55, 14.10.1-14.10.91. https://doi.org/10.1002/cpbi.11

[34] Akhkha, A., Tahar, B., Hazem, K., Parvaiz, A. and Piotr, D. (2013) Chlorophyll Fluorescence: A Potential Selection Criterion for Drought Tolerance in Selected Durum Wheat (Triticum durum Desf.) Cultivars. SOAJ NanoPhotoBioSciences, 1, 147-156.

[35] Ahmed, M. and Fayyaz-Ul-Hassan. (2015) Response of Spring Wheat (Triticum aestivum L.) Quality Traits and Yield to Sowing Date. PLoS ONE, 10, e0126097.

[36] Silvente, S., Sobolev, A.P. and Lara, M. (2012) Metabolite Adjustments in Drought Tolerant and Sensitive Soybean Genotypes in Response to Water Stress. PLoS ONE, 7, e38554. https://doi.org/10.1371/journal.pone.0038554

[37] Krasensky, J. and Jonak, C. (2012) Drought, Salt, and Temperature Stress-Induced Metabolic Rearrangements and Regulatory Networks. Journal of Experimental Botany, 63, 1593-1608. https://doi.org/10.1093/jxb/err460

[38] Rai, K. (2002) Role of Amino Acid in Plant Responses to Stresses. Biologia Plantarum, 45, 481-487. https://doi.org/10.1023/A:1022308229759

[39] Rontein, D., Basset, G. and Hanson, A.D. (2002) Metabolic Engineering of Osmoprotectant Accumulation in Plants. Metabolic Engineering, 4, 49-56. https://doi.org/10.1006/mben.2001.0208

[40] Krugman, T., Peleg, Z., Quansah, L., Chagué, V., Korol, A.B., Nevo, E., Saranga, Y., Fait, A., Chalhoub, B. and Fahima, T. (2011) Alteration in Expression of Hormone-Related Genes in Wild Emmer Wheat Roots Associated with Drought Adaptation Mechanisms. Functional \& Integrative Genomics, 11, 565-583. https://doi.org/10.1007/s10142-011-0231-6

[41] Witt, S., Galicia, L., Lisec, J., Cairns, J., Tiessen, A., Araus, J.L., Palacios-Rojas, N. and Fernie, A.R. (2012) Metabolic and Phenotypic Responses of Greenhouse-Grown Maize Hybrids to Experimentally Controlled Drought Stress. Molecular Plant, 5, 401-417. https://doi.org/10.1093/mp/ssr102

[42] Herrmann, K.M. and Weaver, L.M. (1999) The Shikimate Pathway. Annual Review of Plant Biology, 50, 473-503. https://doi.org/10.1146/annurev.arplant.50.1.473

[43] Maeda, H. and Dudareva, N. (2012) The Shikimate Pathway and Aromatic Amino Acid Biosynthesis in Plants. Annual Review of Plant Biology, 63, 73-105. https://doi.org/10.1146/annurev-arplant-042811-105439

[44] Suguiyama, V.F., Silva, E.A., Meirelles, S.T., Centeno, D.C. and Braga, M.R. (2014) Leaf Metabolite Profile of the Brazilian Resurrection Plant Barbacenia purpurea Hook. (Velloziaceae) Shows Two Time-Dependent Responses during Desiccation and Recovering. Frontiers in Plant Science, 5, 96. https://doi.org/10.3389/fpls.2014.00096

[45] Ramanjulu, S. and Sudhakar, C. (2000) Proline Metabolism during Dehydration in 
Two Mulberry Genotypes with Contrasting Drought Tolerance. Journal of Plant Physiology, 157, 81-85. https://doi.org/10.1016/S0176-1617(00)80139-X

[46] Kumar, S.G., Reddy, A.M. and Sudhakar, C. (2003) NaCl Effects on Proline Metabolism in Two High Yielding Genotypes of Mulberry (Morusalba L.) with Contrasting Salt Tolerance. Plant Science, 165, 1245-1251.

https://doi.org/10.1016/S0168-9452(03)00332-7

[47] Hayat, S., Hayat, Q., Alyemeni, M.N., Wani, A.S., Pichtel, J. and Ahmad, A. (2012) Role of Proline under Changing Environments. Plant Signaling \& Behavior, 7, 1456-1466. https://doi.org/10.4161/psb.21949

[48] Shao, H.-B., Chen, X.-Y., Chu, L.-Y., Zhao, X.-N., Wu, G., Yuan, Y.-B., Zhao, C.-X. and $\mathrm{Hu}, \mathrm{Z} . \mathrm{-M}$. (2006) Investigation on the Relationship of Proline with Wheat Anti-Drought under Soil Water Deficits. Colloids and Surfaces B: Biointerfaces, 53, 113-119. https://doi.org/10.1016/j.colsurfb.2006.08.008

[49] Hare, P.D., Cress, W.A. and Van Staden, J. (1998) Dissecting the Roles of Osmolyte Accumulation during Stress. Plant, Cell \& Environment, 21, 535-553. https://doi.org/10.1046/j.1365-3040.1998.00309.x

[50] Malatrasi, M., Corradi, M., Svensson, J.T., Close, T.J., Gulli, M. and Marmiroli, N. (2006) A Branched-Chain Amino Acid Aminotransferase Gene Isolated from Hordeum vulgare Is Differentially Regulated by Drought Stress. Theoretical and Applied Genetics, 113, 965-976. https://doi.org/10.1007/s00122-006-0339-6

[51] Rizhsky, L., Liang, H., Shuman, J., Shulaev, V., Davletova, S. and Mittler, R. (2004) When Defense Pathways Collide: The Response of Arabidopsis to a Combination of Drought and Heat Stress. Plant Physiology, 134, 1683-1696.

https://doi.org/10.1104/pp.103.033431

[52] Taylor, N.L., Heazlewood, J.L., Day, D.A. and Millar, A.H. (2004) Lipoic Acid-Dependent Oxidative Catabolism of A-Keto Acids in Mitochondria Provides Evidence for Branched-Chain Amino Acid Catabolism in Arabidopsis. Plant Physiology, 134, 838-848. https://doi.org/10.1104/pp.103.035675

[53] Templer, S.E., Ammon, A., Pscheidt, D., Ciobotea, O., Schuy, C., McCollum, C., Sonnewald, U., Hanemann, A., Förster, J., Ordon, F., von Korff, M. and Voll, L.M. (2017) Metabolite Profiling of Barley Flag Leaves under Drought and Combined Heat and Drought Stress Reveals Metabolic QTLs for Metabolites Associated with Antioxidant Defense. Journal of Experimental Botany, 68, 1697-1713.

https://doi.org/10.1093/jxb/erx038

[54] Garg, A.K., Kim, J.K., Owens, T.G., Ranwala, A.P., Do Choi, Y., Kochian, L.V. and Wu, R.J. (2002) Trehalose Accumulation in Rice Plants Confers High Tolerance Levels to Different Abiotic Stresses. Proceedings of the National Academy of Sciences of the United States of America, 99, 15898-15903.

https://doi.org/10.1073/pnas.252637799

[55] Paul, M.J., Primavesi, L.F., Jhurreea, D. and Zhang, Y. (2008) Trehalose Metabolism and Signaling. Annual Review of Plant Biology, 59, 417-441. https://doi.org/10.1146/annurev.arplant.59.032607.092945

[56] Van den Ende, W. (2013) Multifunctional Fructans and Raffinose Family Oligosaccharides. Frontiers in Plant Science, 4, 247. https://doi.org/10.3389/fpls.2013.00247

[57] Servillo, L., Giovane, A., Balestrieri, M.L., Ferrari, G., Cautela, D. and Castaldo, D. (2012) Occurrence of Pipecolic Acid and Pipecolic Acid Betaine (Homostachydrine) in Citrus Genus Plants. Journal of Agricultural and Food Chemistry, 60, 315-321. https://doi.org/10.1021/jf204286r

[58] Siritunga, D. and Sayre, R. (2004) Engineering Cyanogen Synthesis and Turnover in 
Cassava (Manihot esculenta). Plant Molecular Biology, 56, 661-669.

https://doi.org/10.1007/s11103-004-3415-9

[59] Burns, A., Gleadow, R., Cliff, J., Zacarias, A. and Cavagnaro, T. (2010) Cassava: The Drought, War and Famine Crop in a Changing World. Sustainability, 2, 3572-3607. https://doi.org/10.3390/su2113572

[60] Bénard, C., Bernillon, S., Biais, B., Osorio, S., Maucourt, M., Ballias, P., Deborde, C., Colombié, S., Cabasson, C., Jacob, D., Vercambre, G., Gautier, H., Rolin, D., Genard, M., Fernie, A.R., Gibon, Y. and Moing, A. (2015) Metabolomic Profiling in Tomato Reveals Diel Compositional Changes in Fruit Affected by Source-Sink Relationships. Journal of Experimental Botany, 66, 3391-3404.

https://doi.org/10.1093/jxb/erv151

[61] Tzin, V. and Galili, G. (2010) New Insights into the Shikimate and Aromatic Amino Acids Biosynthesis Pathways in Plants. Molecular Plant, 3, 956-972.

https://doi.org/10.1093/mp/ssq048

[62] Wang, M., Ma, X., Shen, J., Li, C. and Zhang, W. (2014) The Ongoing Story: The Mitochondria Pyruvate Carrier 1 in Plant Stress Response in Arabidopsis. Plant Signaling \& Behavior, 9, e973810. https://doi.org/10.4161/15592324.2014.973810

[63] Verslues, P.E. and Sharma, S. (2010) Proline Metabolism and Its Implications for Plant-Environment Interaction. The Arabidopsis Book, 8, e0140.

https://doi.org/10.1199/tab.0140

[64] Hare, P.D. and Cress, W.A. (1997) Metabolic Implications of Stress-Induced Proline Accumulation in Plants. Plant Growth Regulation, 21, 79-102.

https://doi.org/10.1023/A:1005703923347 
Supplemental Table 1. List of significant metabolites with their compound type and identifier (KEGG ID/PubChemCID $\left({ }^{*}\right) /$ ChEBI ID/UNDP ID), molecular formula (MF), mass-to-charge ratio (m/z), retention time (RT), P-value, false discovery rate $($ FDR) and fold change (Drought/Control, D/C). (KEGG = Kyoto Encyclopedia of Genes and Genomes, ChEBI = Chemical Entities of Biological Interest, UNDP = United Natural Products Database).

\begin{tabular}{|c|c|c|c|c|c|c|c|c|c|c|}
\hline Sl\# & Compound name & $\begin{array}{l}\text { Compound } \\
\text { ID }\end{array}$ & MF & $\begin{array}{l}\text { Compound } \\
\text { type }\end{array}$ & $\mathrm{m} / \mathrm{z}$ & RT & t.stat & p. value & FDR & $\begin{array}{l}\text { Fold Change } \\
\text { (D/C) }\end{array}$ \\
\hline & \multicolumn{10}{|l|}{$\begin{array}{l}\text { Amino acids and } \\
\text { their derivatives }\end{array}$} \\
\hline 1 & $\begin{array}{l}\text { 4-Aminobutanoate } \\
\text { (GABA) }\end{array}$ & $\mathrm{C} 00334$ & $\mathrm{C}_{4} \mathrm{H}_{9} \mathrm{NO}_{2}$ & Amino acid & 102.0562 & 0.82 & 6.0031 & $2.87 \mathrm{E}-07$ & $1.72 \mathrm{E}-06$ & -1.92 \\
\hline 2 & 5-oxo-L-proline & C01879 & $\mathrm{C}_{5} \mathrm{H}_{7} \mathrm{NO}_{3}$ & Amino acid & 130.0503 & 1.84 & 2.4005 & 0.020473 & 0.030014 & -1.14 \\
\hline 3 & Clithioneine & UNPD118571 & $\mathrm{C}_{13} \mathrm{H}_{22} \mathrm{~N}_{4} \mathrm{O}_{5} \mathrm{~S}$ & $\begin{array}{l}\text { Amino acid } \\
\text { derivative }\end{array}$ & 347.1313 & 6.27 & 6.0628 & $2.33 \mathrm{E}-07$ & $1.44 \mathrm{E}-06$ & -1.71 \\
\hline 4 & Glutathione & $\mathrm{C} 00051$ & $\mathrm{C}_{10} \mathrm{H}_{17} \mathrm{~N}_{3} \mathrm{O}_{6} \mathrm{~S}$ & Amino acid & 306.0758 & 1.61 & 3.4984 & 0.0010502 & 0.002194 & -2.29 \\
\hline 6 & L-Carnitine & $\mathrm{C} 00318$ & $\mathrm{C}_{7} \mathrm{H}_{15} \mathrm{NO}_{3}$ & $\begin{array}{l}\text { Amino acid } \\
\text { derivative }\end{array}$ & 162.1124 & 1.28 & 7.9845 & $3.12 \mathrm{E}-10$ & $6.55 \mathrm{E}-09$ & -2.01 \\
\hline 7 & L-glutamine & C00303 & $\mathrm{C}_{5} \mathrm{H}_{10} \mathrm{~N}_{2} \mathrm{O}_{3}$ & Amino acid & 145.0617 & 0.73 & 4.6058 & $3.26 \mathrm{E}-05$ & $9.37 \mathrm{E}-05$ & -1.93 \\
\hline 8 & L-Isoleucine & C16434 & $\mathrm{C}_{6} \mathrm{H}_{13} \mathrm{NO}_{2}$ & Amino acid & 132.1021 & 2.44 & -2.6682 & 0.010495 & 0.017219 & 1.47 \\
\hline 9 & L-Phenylalanine & $\mathrm{C} 00079$ & $\mathrm{C}_{9} \mathrm{H}_{11} \mathrm{NO}_{2}$ & Amino acid & 164.0709 & 5.52 & -3.013 & 0.0041963 & 0.007468 & 1.77 \\
\hline 10 & L-Proline & $\mathrm{C} 00148$ & $\mathrm{C}_{5} \mathrm{H}_{9} \mathrm{NO}_{2}$ & Amino acid & 116.0706 & 0.89 & -6.332 & $9.19 \mathrm{E}-08$ & $6.23 \mathrm{E}-07$ & 3.90 \\
\hline 11 & L-Serine & C00065 & $\mathrm{C}_{3} \mathrm{H}_{7} \mathrm{NO}_{3}$ & Amino acid & 104.035 & 0.75 & 4.9825 & $9.32 \mathrm{E}-06$ & $3.32 \mathrm{E}-05$ & -2.36 \\
\hline 12 & L-Threonine & $\mathrm{C} 00188$ & $\mathrm{C}_{4} \mathrm{H}_{9} \mathrm{NO}_{3}$ & Amino acid & 118.0508 & 0.76 & 5.4282 & $2.06 \mathrm{E}-06$ & $9.02 \mathrm{E}-06$ & -1.90 \\
\hline 15 & N-hydroxy-L-valine & C20313 & $\mathrm{C}_{5} \mathrm{H}_{11} \mathrm{NO}_{3}$ & $\begin{array}{l}\text { Amino acid } \\
\text { derivative }\end{array}$ & 134.0817 & 0.93 & 4.1862 & 0.0001266 & 0.000332 & -1.73 \\
\hline 16 & Oxidized glutathione & $\mathrm{C} 00127$ & $\mathrm{C}_{20} \mathrm{H}_{32} \mathrm{~N}_{6} \mathrm{O}_{12} \mathrm{~S}_{2}$ & Amino acid & 611.1441 & 3.74 & 6.6817 & $2.74 \mathrm{E}-08$ & $2.21 \mathrm{E}-07$ & -2.21 \\
\hline 17 & $\begin{array}{l}\text { S-phospho-L-cysteine(2-) } \\
\text { residue }\end{array}$ & CHEBI: 61975 & $\mathrm{C}_{3} \mathrm{H}_{4} \mathrm{NO}_{4} \mathrm{PS}$ & $\begin{array}{l}\text { Amino acid } \\
\text { residue }\end{array}$ & 181.9621 & 0.69 & 4.4705 & $5.07 \mathrm{E}-05$ & 0.00014 & -1.76 \\
\hline \multirow[t]{2}{*}{18} & Tyrosine & C01536 & $\mathrm{C}_{9} \mathrm{H}_{11} \mathrm{NO}_{3}$ & Amino acid & 182.0819 & 3.16 & -2.4198 & 0.019539 & 0.0291 & 1.61 \\
\hline & $\begin{array}{l}\text { Sugars and their } \\
\text { derivatives }\end{array}$ & & & & & & & & & \\
\hline 19 & D-(-)-Arabinose & $\mathrm{C} 00216$ & $\mathrm{C}_{5} \mathrm{H}_{10} \mathrm{O}_{5}$ & Sugar & 149.0448 & 0.83 & 6.6977 & $2.59 \mathrm{E}-08$ & $2.18 \mathrm{E}-07$ & -1.75 \\
\hline 20 & D-(+)-Raffinose & C00492 & $\mathrm{C}_{18} \mathrm{H}_{32} \mathrm{O}_{16}$ & Sugar & 527.1589 & 0.97 & 5.345 & $2.74 \mathrm{E}-06$ & $1.13 \mathrm{E}-05$ & -1.97 \\
\hline 21 & D-(+)-Trehalose & C01083 & $\mathrm{C}_{12} \mathrm{H}_{22} \mathrm{O}_{11}$ & Sugar & 377.0853 & 0.92 & 7.4476 & $1.95 \mathrm{E}-09$ & $2.95 \mathrm{E}-08$ & -2.07 \\
\hline 22 & $\begin{array}{l}\text { Decyl beta-D- } \\
\text { maltopyranoside }\end{array}$ & CHEBI: 67097 & $\mathrm{C}_{22} \mathrm{H}_{42} \mathrm{O}_{11}$ & Sugar & 481.2579 & 11.56 & 2.2757 & 0.027565 & 0.039648 & -1.24 \\
\hline 23 & Deoxyribose & $\mathrm{C} 01801$ & $\mathrm{C}_{5} \mathrm{H}_{10} \mathrm{O}_{4}$ & Sugar & 133.0511 & 1.82 & 3.7356 & 0.0005156 & 0.001152 & -2.71 \\
\hline
\end{tabular}




\section{Continued}

\begin{tabular}{|c|c|c|c|c|c|c|c|c|c|c|}
\hline 24 & D-Glucosamine & C00329 & $\mathrm{C}_{6} \mathrm{H}_{13} \mathrm{NO}_{5}$ & Sugar & 180.087 & 0.84 & 2.9512 & 0.0049675 & 0.008621 & -1.37 \\
\hline 25 & D-Glucose & $\mathrm{C} 00031$ & $\mathrm{C}_{6} \mathrm{H}_{12} \mathrm{O}_{6}$ & Sugar & 179.055 & 0.82 & 2.3984 & 0.020581 & 0.030014 & -1.35 \\
\hline 26 & D-Threo-L-galacto-octose & $16019992^{*}$ & $\mathrm{C}_{8} \mathrm{H}_{16} \mathrm{O}_{8}$ & Sugar & 239.0763 & 0.89 & 7.7616 & $6.66 \mathrm{E}-10$ & $1.27 \mathrm{E}-08$ & -1.82 \\
\hline 27 & Lichenin & $\mathrm{C} 00478$ & $\mathrm{C}_{6} \mathrm{H}_{10} \mathrm{O}_{5}$ & Sugar & 161.0444 & 0.8 & 3.0431 & 0.0038627 & 0.006933 & -1.69 \\
\hline 28 & N-Acetyl-D-Fucosamine & C15480 & $\mathrm{C}_{8} \mathrm{H}_{15} \mathrm{NO}_{5}$ & Sugar & 206.1027 & 1.46 & 5.0824 & $6.66 \mathrm{E}-06$ & $2.50 \mathrm{E}-05$ & -2.30 \\
\hline 29 & Sedoheptulose & C02076 & $\mathrm{C}_{7} \mathrm{H}_{14} \mathrm{O}_{7}$ & Sugar & 209.0657 & 0.85 & 5.1809 & $4.78 \mathrm{E}-06$ & $1.89 \mathrm{E}-05$ & -1.64 \\
\hline 30 & Sucrose & C00089 & $\mathrm{C}_{12} \mathrm{H}_{22} \mathrm{O}_{11}$ & Sugar & 365.1054 & 0.93 & 6.95 & $1.08 \mathrm{E}-08$ & $1.14 \mathrm{E}-07$ & -1.96 \\
\hline 31 & $\begin{array}{l}\text { Alpha-Methylene } \\
\text { Gamma-butyrolactone }\end{array}$ & $68352^{*}$ & $\mathrm{C}_{5} \mathrm{H}_{6} \mathrm{O}_{2}$ & Sugar acid & 99.0441 & 0.91 & 5.5991 & $1.15 \mathrm{E}-06$ & $5.62 \mathrm{E}-06$ & -1.97 \\
\hline 32 & D-Arabinono-1,4-lactone & C00652 & $\mathrm{C}_{5} \mathrm{H}_{8} \mathrm{O}_{5}$ & Sugar acid & 147.0291 & 1.49 & 2.1823 & 0.034229 & 0.048898 & -1.10 \\
\hline 33 & D-Glucono-1,5-lactone & C00198 & $\mathrm{C}_{6} \mathrm{H}_{10} \mathrm{O}_{6}$ & Sugar acid & 177.0396 & 0.96 & 3.4477 & 0.0012195 & 0.002486 & -1.93 \\
\hline 34 & D-xylonate & C00502 & $\mathrm{C}_{5} \mathrm{H}_{10} \mathrm{O}_{6}$ & Sugar acid & 195.0504 & 0.75 & 9.3838 & $2.96 \mathrm{E}-12$ & $1.55 \mathrm{E}-10$ & -2.06 \\
\hline 35 & Threonate & $\mathrm{C} 01620$ & $\mathrm{C}_{4} \mathrm{H}_{8} \mathrm{O}_{5}$ & Sugar acid & 135.0298 & 0.81 & 2.0257 & 0.048623 & 0.067176 & -1.09 \\
\hline 36 & $\begin{array}{l}\text { 6-(a-D-glucosaminyl)-1D- } \\
\text { myo-inositol (GlcN)1 (Ino)1 }\end{array}$ & C15658 & $\mathrm{C}_{12} \mathrm{H}_{23} \mathrm{NO}_{10}$ & Sugar alcohol & 342.1388 & 0.84 & 7.1051 & $6.34 \mathrm{E}-09$ & $7.40 \mathrm{E}-08$ & -2.90 \\
\hline 37 & $\begin{array}{l}\text { Bis-D-fructose } \\
\text { 2',1:2,1'-dianhydride }\end{array}$ & $\mathrm{C} 04333$ & $\mathrm{C}_{12} \mathrm{H}_{20} \mathrm{O}_{10}$ & $\begin{array}{c}\text { Sugar } \\
\text { dianhydride }\end{array}$ & 325.113 & 0.91 & 7.1369 & $5.68 \mathrm{E}-09$ & $7.02 \mathrm{E}-08$ & -2.36 \\
\hline \multicolumn{11}{|c|}{ Amines } \\
\hline 38 & Piperidine & C01746 & $\mathrm{C}_{5} \mathrm{H}_{11} \mathrm{~N}$ & Amine & 86.0966 & 2.45 & -2.6375 & 0.011354 & 0.018483 & 1.50 \\
\hline 39 & Spermidine & $\mathrm{C} 00315$ & $\mathrm{C}_{7} \mathrm{H}_{19} \mathrm{~N}_{3}$ & Amine & 146.1657 & 0.58 & 5.7159 & $7.70 \mathrm{E}-07$ & $3.95 \mathrm{E}-06$ & -1.90 \\
\hline \multicolumn{11}{|c|}{ Fatty acids } \\
\hline 40 & 2-Hydroxyheptanoic acid & $2750949^{\star}$ & $\mathrm{C}_{7} \mathrm{H}_{14} \mathrm{O}_{3}$ & Fatty acid & 145.086 & 8.05 & 5.4186 & $2.13 \mathrm{E}-06$ & $9.14 \mathrm{E}-06$ & -1.85 \\
\hline 41 & Linoleate & C01595 & $\mathrm{C}_{18} \mathrm{H}_{32} \mathrm{O}_{2}$ & Fatty acid & 279.2313 & 14.58 & 3.6415 & 0.0006853 & 0.001499 & -1.79 \\
\hline 42 & Palmitoleic acid & $\mathrm{C} 08362$ & $\mathrm{C}_{16} \mathrm{H}_{30} \mathrm{O}_{2}$ & Fatty acid & 253.216 & 14.89 & 4.8821 & $1.30 \mathrm{E}-05$ & $4.35 \mathrm{E}-05$ & -2.10 \\
\hline \multicolumn{11}{|c|}{ Nucleosides/Nucleobases } \\
\hline 43 & Adenine & $\mathrm{C} 00147$ & $\mathrm{C}_{5} \mathrm{H}_{5} \mathrm{~N}_{5}$ & Nucleobase & 134.0462 & 5.31 & 4.4942 & $4.69 \mathrm{E}-05$ & 0.000131 & -1.63 \\
\hline 44 & Adenosine & $\mathrm{C} 00212$ & $\mathrm{C}_{10} \mathrm{H}_{13} \mathrm{~N}_{5} \mathrm{O}_{4}$ & Nucleoside & 268.1043 & 5.31 & 3.6589 & 0.0006504 & 0.001438 & -1.51 \\
\hline 45 & Deoxyadenosine & C00559 & $\mathrm{C}_{10} \mathrm{H}_{13} \mathrm{~N}_{5} \mathrm{O}_{3}$ & Nucleoside & 252.1093 & 5.37 & 2.5426 & 0.014433 & 0.022618 & -1.36 \\
\hline 46 & Guanine & $\mathrm{C} 00242$ & $\mathrm{C}_{5} \mathrm{H}_{5} \mathrm{~N}_{5} \mathrm{O}$ & Nucleobase & 152.0569 & 5.43 & 2.4568 & 0.017852 & 0.02697 & -1.34 \\
\hline 47 & Guanosine & $\mathrm{C} 00387$ & $\mathrm{C}_{10} \mathrm{H}_{13} \mathrm{~N}_{5} \mathrm{O}_{5}$ & Nucleoside & 282.0831 & 5.4 & 3.0836 & 0.003453 & 0.006361 & -1.30 \\
\hline 48 & Uracil & C00106 & $\mathrm{C}_{4} \mathrm{H}_{4} \mathrm{~N}_{2} \mathrm{O}_{2}$ & Nucleobase & 113.0347 & 2.73 & 2.9045 & 0.0056354 & 0.009621 & -1.12 \\
\hline 49 & Uridine & C00299 & $\mathrm{C}_{9} \mathrm{H}_{12} \mathrm{~N}_{2} \mathrm{O}_{6}$ & Nucleoside & 243.0619 & 2.73 & 2.3685 & 0.022118 & 0.032033 & -1.07 \\
\hline \multicolumn{11}{|c|}{ Organic acids } \\
\hline 50 & 12-Hydroxyjasmonic acid & C21385 & $\mathrm{C}_{12} \mathrm{H}_{18} \mathrm{O}_{4}$ & Organic acid & 227.1284 & 8.66 & 5.843 & $4.98 \mathrm{E}-07$ & $2.82 \mathrm{E}-06$ & -2.37 \\
\hline 51 & $\begin{array}{l}\text { 2-(Acetamidomethylene) } \\
\text { succinate }\end{array}$ & $\mathrm{C} 01215$ & $\mathrm{C}_{7} \mathrm{H}_{9} \mathrm{NO}_{5}$ & Organic acid & 188.0558 & 2.07 & 4.7742 & $1.87 \mathrm{E}-05$ & $6.03 \mathrm{E}-05$ & -2.09 \\
\hline
\end{tabular}




\section{Continued}

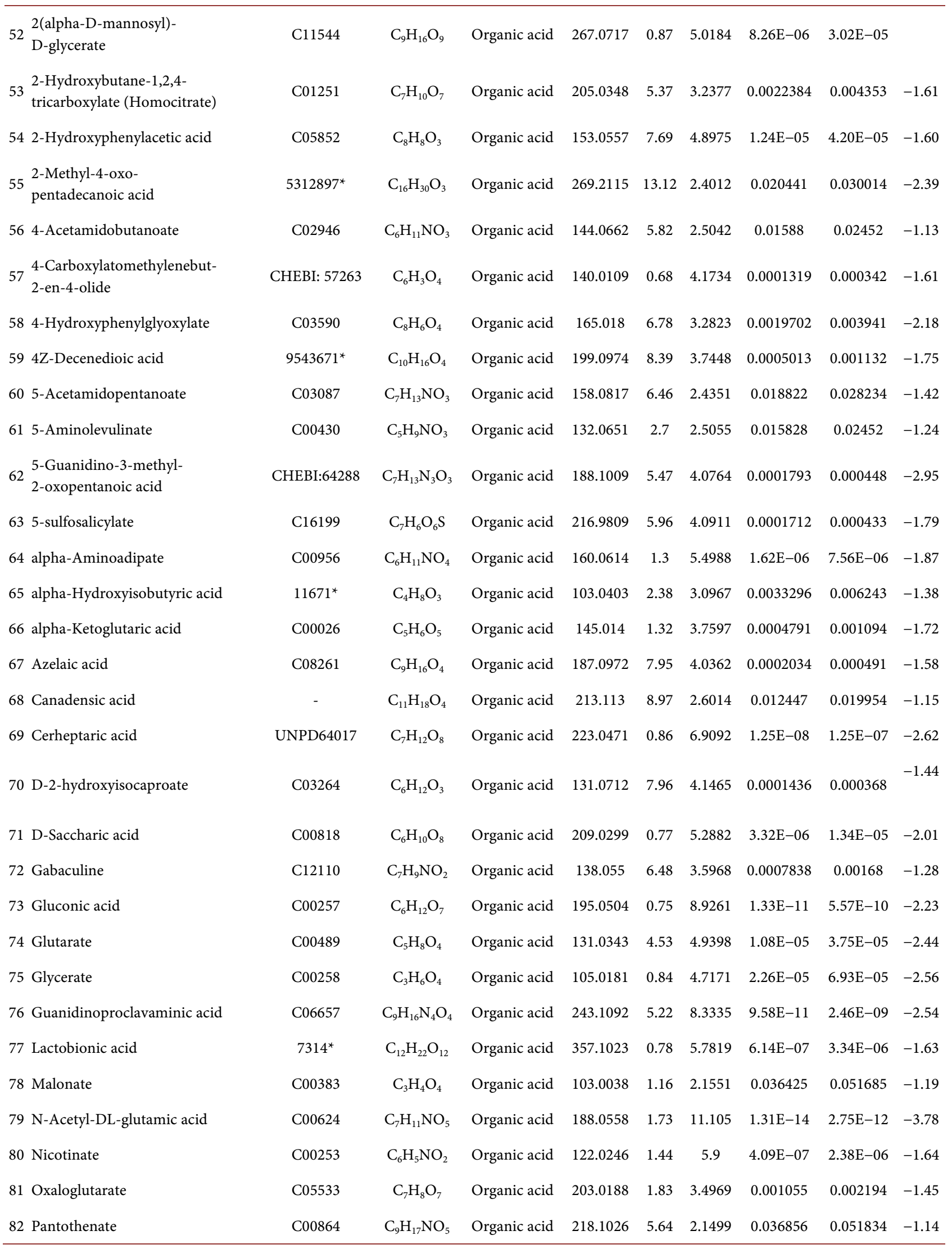




\section{Continued}

\begin{tabular}{|c|c|c|c|c|c|c|c|c|c|c|}
\hline 83 & Phenylpyruvate & C00166 & $\mathrm{C}_{9} \mathrm{H}_{8} \mathrm{O}_{3}$ & Organic acid & 165.0551 & 3.3 & -2.5748 & 0.013313 & 0.021179 & 1.68 \\
\hline 84 & Phosphonatoenolpyruvate & $\mathrm{C} 00074$ & $\mathrm{C}_{3} \mathrm{H}_{2} \mathrm{O}_{6} \mathrm{P}$ & Organic acid & 165.9665 & 0.72 & 5.0944 & $6.40 \mathrm{E}-06$ & $2.44 \mathrm{E}-05$ & -2.02 \\
\hline 85 & Pipecolate & C00408 & $\mathrm{C}_{6} \mathrm{H}_{11} \mathrm{NO}_{2}$ & Organic acid & 130.0864 & 1.28 & -4.5763 & $3.59 \mathrm{E}-05$ & 0.000102 & 2.17 \\
\hline 86 & Pyruvate & C00022 & $\mathrm{C}_{3} \mathrm{H}_{4} \mathrm{O}_{3}$ & Organic acid & 87.0085 & 1.31 & 5.7788 & $6.21 \mathrm{E}-07$ & $3.34 \mathrm{E}-06$ & -1.63 \\
\hline 87 & Quinate & C00296 & $\mathrm{C}_{7} \mathrm{H}_{12} \mathrm{O}_{6}$ & Organic acid & 191.055 & 0.86 & 4.6342 & $2.97 \mathrm{E}-05$ & $8.77 \mathrm{E}-05$ & -2.50 \\
\hline 88 & Sebacic acid & C08277 & $\mathrm{C}_{10} \mathrm{H}_{18} \mathrm{O}_{4}$ & Organic acid & 201.1126 & 8.57 & 4.4566 & $5.30 \mathrm{E}-05$ & 0.000145 & -1.85 \\
\hline 89 & Shikimate & C00493 & $\mathrm{C}_{7} \mathrm{H}_{10} \mathrm{O}_{5}$ & Organic acid & 173.0448 & 1.11 & 2.6138 & 0.012061 & 0.019484 & 1.21 \\
\hline \multirow[t]{2}{*}{90} & Suberic acid & C08278 & $\mathrm{C}_{8} \mathrm{H}_{14} \mathrm{O}_{4}$ & Organic acid & 173.0816 & 7.63 & 4.0376 & 0.0002026 & 0.000491 & -1.57 \\
\hline & Organic compounds & & & & & & & & & \\
\hline 91 & $\begin{array}{l}\text { 1-(Indol-3-yl)propanol } \\
\text { 3-phosphate }\end{array}$ & C04229 & $\mathrm{C}_{11} \mathrm{H}_{14} \mathrm{NO}_{4} \mathrm{P}$ & $\begin{array}{l}\text { Phosphorylated } \\
\text { compound }\end{array}$ & 256.0813 & 1.37 & 6.7493 & $2.17 \mathrm{E}-08$ & $1.98 \mathrm{E}-07$ & -1.98 \\
\hline 92 & Fructoselysine 6-phosphate & C16489 & $\mathrm{C}_{12} \mathrm{H}_{25} \mathrm{~N}_{2} \mathrm{O}_{10} \mathrm{P}$ & $\begin{array}{l}\text { Phosphorylated } \\
\text { compound }\end{array}$ & 387.1141 & 1.04 & 7.0276 & $8.28 \mathrm{E}-09$ & $9.15 \mathrm{E}-08$ & -1.96 \\
\hline 93 & 1,2,3-Trihydroxybenzene & C01108 & $\mathrm{C}_{6} \mathrm{H}_{6} \mathrm{O}_{3}$ & $\begin{array}{l}\text { Organic } \\
\text { compound }\end{array}$ & 127.0394 & 5.31 & 5.5453 & $1.38 \mathrm{E}-06$ & $6.60 \mathrm{E}-06$ & -1.78 \\
\hline 94 & 2-Aminophenol & C01987 & $\mathrm{C}_{6} \mathrm{H}_{7} \mathrm{NO}$ & $\begin{array}{l}\text { Organic } \\
\text { compound }\end{array}$ & 110.0603 & 2.14 & 2.9358 & 0.0051793 & 0.008915 & -1.48 \\
\hline 95 & 3-Indoleacetonitrile & C02938 & $\mathrm{C}_{10} \mathrm{H}_{8} \mathrm{~N}_{2}$ & $\begin{array}{l}\text { Organic } \\
\text { compound }\end{array}$ & 157.085 & 5.82 & 3.3726 & 0.0015182 & 0.003066 & -2.07 \\
\hline 96 & 3-tert-Butyl-5-methylcatechol & C03929 & $\mathrm{C}_{11} \mathrm{H}_{16} \mathrm{O}_{2}$ & $\begin{array}{l}\text { Organic } \\
\text { compound }\end{array}$ & 181.1222 & 10.08 & 4.9364 & $1.09 \mathrm{E}-05$ & $3.75 \mathrm{E}-05$ & -1.66 \\
\hline 97 & 4-Hydroxybenzoate & C00156 & $\mathrm{C}_{7} \mathrm{H}_{6} \mathrm{O}_{3}$ & $\begin{array}{l}\text { Organic } \\
\text { compound }\end{array}$ & 137.0235 & 8.66 & 3.7608 & 0.0004774 & 0.001094 & -1.95 \\
\hline 98 & 4-Nitroacetophenone & C02803 & $\mathrm{C}_{8} \mathrm{H}_{7} \mathrm{NO}_{3}$ & $\begin{array}{l}\text { Organic } \\
\text { compound }\end{array}$ & 166.058 & 7.19 & 4.7636 & $1.93 \mathrm{E}-05$ & $6.15 \mathrm{E}-05$ & -1.76 \\
\hline 99 & 5-Hydroxyisourate & C11821 & $\mathrm{C}_{5} \mathrm{H}_{4} \mathrm{~N}_{4} \mathrm{O}_{4}$ & $\begin{array}{l}\text { Organic } \\
\text { compound }\end{array}$ & 185.032 & 0.74 & 3.8129 & 0.000407 & 0.00095 & -1.66 \\
\hline 100 & $\begin{array}{l}\text { 5'-O-beta-D- } \\
\text { Glucosylpyridoxine }\end{array}$ & C03996 & $\mathrm{C}_{14} \mathrm{H}_{21} \mathrm{NO}_{8}$ & $\begin{array}{l}\text { Organic } \\
\text { compound }\end{array}$ & 332.1333 & 4.05 & 8.5242 & $5.05 \mathrm{E}-11$ & $1.77 \mathrm{E}-09$ & -2.00 \\
\hline 101 & alpha-Sinensal & C09729 & $\mathrm{C}_{15} \mathrm{H}_{22} \mathrm{O}$ & $\begin{array}{l}\text { Organic } \\
\text { compound }\end{array}$ & 219.1748 & 12.13 & 4.2018 & 0.0001205 & 0.00032 & -2.19 \\
\hline 102 & Asperuloside & C09769 & $\mathrm{C}_{18} \mathrm{H}_{22} \mathrm{O}_{11}$ & $\begin{array}{l}\text { Organic } \\
\text { compound }\end{array}$ & 413.1083 & 6.91 & 5.4836 & $1.71 \mathrm{E}-06$ & $7.79 \mathrm{E}-06$ & -1.84 \\
\hline 103 & $\begin{array}{l}\text { Aurantio-obtusin } \\
\text { beta-D-glucoside }\end{array}$ & C10303 & $\mathrm{C} 23 \mathrm{H} 24 \mathrm{O} 12$ & $\begin{array}{l}\text { Organic } \\
\text { compound }\end{array}$ & 493.1328 & 8.2 & 4.7143 & $2.28 \mathrm{E}-05$ & $6.93 \mathrm{E}-05$ & -1.55 \\
\hline 104 & Benzoate & C00180 & $\mathrm{C}_{7} \mathrm{H}_{6} \mathrm{O}_{2}$ & $\begin{array}{l}\text { Organic } \\
\text { compound }\end{array}$ & 121.0291 & 7.61 & 3.6032 & 0.0007688 & 0.001664 & -1.80 \\
\hline 105 & beta-Cymaropyranose & C08234 & $\mathrm{C}_{7} \mathrm{H}_{14} \mathrm{O}_{4}$ & $\begin{array}{l}\text { Organic } \\
\text { compound }\end{array}$ & 163.097 & 6.27 & 5.3973 & $2.29 \mathrm{E}-06$ & $9.63 \mathrm{E}-06$ & -2.78 \\
\hline 106 & beta-Damascenone & CHEBI: 67251 & $\mathrm{C}_{13} \mathrm{H}_{18} \mathrm{O}$ & $\begin{array}{l}\text { Organic } \\
\text { compound }\end{array}$ & 191.1437 & 8.43 & 4.2446 & 0.0001051 & 0.000283 & -1.42 \\
\hline
\end{tabular}




\section{Continued}

\begin{tabular}{|c|c|c|c|c|c|c|c|c|c|c|}
\hline 107 & Calystegin B2 & C10851 & $\mathrm{C}_{7} \mathrm{H}_{13} \mathrm{NO}_{4}$ & $\begin{array}{l}\text { Organic } \\
\text { compound }\end{array}$ & 174.0768 & 2.53 & 5.6104 & $1.11 \mathrm{E}-06$ & $5.53 \mathrm{E}-06$ & -3.08 \\
\hline 108 & Carlinoside & C10026 & $\mathrm{C}_{26} \mathrm{H}_{28} \mathrm{O}_{15}$ & $\begin{array}{l}\text { Organic } \\
\text { compound }\end{array}$ & 579.1354 & 6.83 & 3.4986 & 0.0010497 & 0.002194 & -1.36 \\
\hline 109 & Citrinin & $\mathrm{C} 16765$ & $\mathrm{C}_{13} \mathrm{H}_{14} \mathrm{O}_{5}$ & $\begin{array}{l}\text { Organic } \\
\text { compound }\end{array}$ & 251.0911 & 7.44 & 4.6398 & $2.91 \mathrm{E}-05$ & $8.74 \mathrm{E}-05$ & -1.61 \\
\hline 110 & D-Glucuronolactone & $\mathrm{C} 02670$ & $\mathrm{C}_{6} \mathrm{H}_{10} \mathrm{O}_{7}$ & $\begin{array}{c}\text { Organic } \\
\text { compound }\end{array}$ & 193.0351 & 0.87 & 2.9683 & 0.0047414 & 0.008338 & -1.32 \\
\hline 111 & DIBOA-glucoside & $\mathrm{C} 15772$ & $\mathrm{C}_{14} \mathrm{H}_{17} \mathrm{NO}_{9}$ & $\begin{array}{l}\text { Organic } \\
\text { compound }\end{array}$ & 342.0826 & 5.98 & 9.6755 & $1.15 \mathrm{E}-12$ & $8.05 \mathrm{E}-11$ & -3.28 \\
\hline 113 & $\begin{array}{l}\text { Dihydro-4,4-dimethyl- } \\
\text { 2,3-furandione }\end{array}$ & $\mathrm{C} 01125$ & $\mathrm{C}_{6} \mathrm{H}_{8} \mathrm{O}_{3}$ & $\begin{array}{l}\text { Organic } \\
\text { compound }\end{array}$ & 127.0403 & 6.66 & 3.2591 & 0.0021054 & 0.004171 & -1.85 \\
\hline 114 & Dimethylmaleate & $\mathrm{C} 00922$ & $\mathrm{C}_{6} \mathrm{H}_{8} \mathrm{O}_{4}$ & $\begin{array}{l}\text { Organic } \\
\text { compound }\end{array}$ & 143.0343 & 6.03 & 3.4655 & 0.0011572 & 0.002382 & -1.85 \\
\hline 115 & Eupalitin 3-O-sulfate & $44259774^{*}$ & $\mathrm{C}_{17} \mathrm{H}_{14} \mathrm{O}_{10} \mathrm{~S}$ & $\begin{array}{l}\text { Organic } \\
\text { compound }\end{array}$ & 409.0229 & 8.7 & 7.7014 & $8.18 \mathrm{E}-10$ & $1.43 \mathrm{E}-08$ & -1.96 \\
\hline 116 & Furfural diethyl acetal & C14280 & $\mathrm{C}_{9} \mathrm{H}_{14} \mathrm{O}_{3}$ & $\begin{array}{l}\text { Organic } \\
\text { compound }\end{array}$ & 169.0859 & 9.88 & 3.064 & 0.0036454 & 0.006604 & -1.43 \\
\hline 117 & Ginnalin C & $44512371^{*}$ & $\mathrm{C}_{13} \mathrm{H}_{16} \mathrm{O}_{9}$ & $\begin{array}{l}\text { Organic } \\
\text { compound }\end{array}$ & 315.0719 & 6.05 & 3.2523 & 0.002147 & 0.004214 & -1.65 \\
\hline 118 & Glucogallin & C01158 & $\mathrm{C}_{13} \mathrm{H}_{16} \mathrm{O}_{10}$ & $\begin{array}{c}\text { Organic } \\
\text { compound }\end{array}$ & 331.0673 & 5.67 & 6.1362 & $1.81 \mathrm{E}-07$ & $1.15 \mathrm{E}-06$ & -1.95 \\
\hline 119 & Lilioside A & $101277416^{*}$ & $\mathrm{C}_{11} \mathrm{H}_{20} \mathrm{O}_{9}$ & $\begin{array}{c}\text { Organic } \\
\text { compound }\end{array}$ & 295.1027 & 5.42 & 9.9518 & $4.75 \mathrm{E}-13$ & $4.98 \mathrm{E}-11$ & -2.17 \\
\hline 120 & Linamarin & C01594 & $\mathrm{C}_{10} \mathrm{H}_{17} \mathrm{NO}_{6}$ & $\begin{array}{c}\text { Organic } \\
\text { compound }\end{array}$ & 246.0972 & 6.67 & -5.0156 & $8.34 \mathrm{E}-06$ & $3.02 \mathrm{E}-05$ & 3.31 \\
\hline 121 & Nandrolone & $\mathrm{C} 07254$ & $\mathrm{C}_{18} \mathrm{H}_{26} \mathrm{O}_{2}$ & $\begin{array}{l}\text { Organic } \\
\text { compound }\end{array}$ & 275.2008 & 10.44 & 6.3569 & $8.43 \mathrm{E}-08$ & $5.90 \mathrm{E}-07$ & -2.06 \\
\hline 122 & Nicotinamide & C00153 & $\mathrm{C}_{6} \mathrm{H}_{6} \mathrm{~N}_{2} \mathrm{O}$ & $\begin{array}{c}\text { Organic } \\
\text { compound }\end{array}$ & 123.0553 & 1.67 & 6.7209 & $2.39 \mathrm{E}-08$ & $2.09 \mathrm{E}-07$ & -2.04 \\
\hline 123 & P-Cymene & C06575 & $\mathrm{C}_{10} \mathrm{H}_{14}$ & $\begin{array}{l}\text { Organic } \\
\text { compound }\end{array}$ & 135.1168 & 7.25 & 8.305 & $1.05 \mathrm{E}-10$ & $2.46 \mathrm{E}-09$ & -2.23 \\
\hline 124 & Phytuberin & C09709 & $\mathrm{C}_{17} \mathrm{H}_{26} \mathrm{O}_{4}$ & $\begin{array}{c}\text { Organic } \\
\text { compound }\end{array}$ & 293.1754 & 11.62 & 3.8982 & 0.0003129 & 0.000738 & -1.84 \\
\hline 125 & Propyl cinnamate & C06360 & $\mathrm{C}_{12} \mathrm{H}_{14} \mathrm{O}_{2}$ & $\begin{array}{c}\text { Organic } \\
\text { compound }\end{array}$ & 191.1062 & 8.11 & 3.0877 & 0.0034137 & 0.006344 & -1.42 \\
\hline 126 & Pruyanaside B & UNDP145434 & $\mathrm{C}_{33} \mathrm{H}_{36} \mathrm{O}_{16}$ & $\begin{array}{l}\text { Organic } \\
\text { compound }\end{array}$ & 687.1929 & 8.27 & 7.1476 & $5.48 \mathrm{E}-09$ & $7.02 \mathrm{E}-08$ & -1.98 \\
\hline 127 & Slaframine & $\mathrm{C} 06185$ & $\mathrm{C}_{12} \mathrm{H}_{20} \mathrm{~N}_{2} \mathrm{O}_{3}$ & $\begin{array}{l}\text { Organic } \\
\text { compound }\end{array}$ & 241.1553 & 5.41 & 6.7947 & $1.85 \mathrm{E}-08$ & $1.77 \mathrm{E}-07$ & -1.89 \\
\hline
\end{tabular}




\section{Continued}

128 Strophanthobiose

129 Succinic anhydride

130 Tarennoside

131 Tribenuron methyl

132 Tribufos

\section{Others}

Organic compound

323.135

$6.97 \mathrm{E}-11 \quad 2.09 \mathrm{E}-09 \quad-1.95$

$\mathrm{C} 19524 \quad \mathrm{C}_{4} \mathrm{H}_{4} \mathrm{O}_{3}$

Organic

$101.0234 \quad 2.26 \quad 2.1479$

$0.037024 \quad 0.051834 \quad-1.21$

$\mathrm{C} 11654 \quad \mathrm{C}_{16} \mathrm{H}_{22} \mathrm{O}_{9}$

Organic compound

6.93

4.071

C10962

$\mathrm{C}_{15} \mathrm{H}_{17} \mathrm{~N}_{5} \mathrm{O}_{6} \mathrm{~S}$

Organic compound

396.09

$5125^{*}$

$\mathrm{C}_{12} \mathrm{H}_{27} \mathrm{OPS}_{3}$

Organic compound

$\begin{array}{lll}15.1056 & 5.96 \quad 7.3501\end{array}$

$2.73 \mathrm{E}-09 \quad 3.82 \mathrm{E}-08 \quad-2.29$

\begin{tabular}{|c|c|c|c|c|c|c|c|c|}
\hline $24779499^{*}$ & $\mathrm{C}_{13} \mathrm{H}_{28} \mathrm{NO}_{7} \mathrm{P}$ & Others & 342.1754 & 6.27 & 7.4451 & $1.97 \mathrm{E}-09$ & $2.95 \mathrm{E}-08$ & -2.17 \\
\hline C02796 & $\mathrm{C}_{9} \mathrm{H}_{7} \mathrm{NO}$ & Others & 144.0455 & 8.55 & 3.1847 & 0.0026012 & 0.005012 & -1.66 \\
\hline C02648 & $\mathrm{C}_{4} \mathrm{H}_{4} \mathrm{O}_{2}$ & Others & 85.0283 & 0.91 & 6.1651 & $1.64 \mathrm{E}-07$ & $1.07 \mathrm{E}-06$ & -2.15 \\
\hline C10373 & $\mathrm{C}_{18} \mathrm{H}_{28} \mathrm{O}_{4}$ & Others & 307.1891 & 10.02 & 2.5484 & 0.014227 & 0.022463 & -1.60 \\
\hline UNPD150686 & $\mathrm{C}_{19} \mathrm{H}_{27} \mathrm{NO}_{5}$ & Others & 350.2113 & 8.46 & 5.1702 & $4.95 \mathrm{E}-06$ & $1.93 \mathrm{E}-05$ & -1.80 \\
\hline C03373 & $\mathrm{C}_{8} \mathrm{H}_{14} \mathrm{~N}_{3} \mathrm{O}_{7} \mathrm{P}$ & Others & 296.0659 & 0.77 & 2.825 & 0.0069704 & 0.01171 & -1.53 \\
\hline CHEBI:79258 & $\mathrm{C}_{16} \mathrm{H}_{30} \mathrm{O}_{7}$ & Others & 335.2074 & 9.97 & 2.7686 & 0.0080872 & 0.013479 & -1.91 \\
\hline C11075 & $\mathrm{C}_{12} \mathrm{H}_{16} \mathrm{~N}_{2} \mathrm{O}_{3}$ & Others & 237.124 & 5.42 & 6.4542 & $6.02 \mathrm{E}-08$ & $4.36 \mathrm{E}-07$ & -2.41 \\
\hline $18502^{\star}$ & $\mathrm{C}_{7} \mathrm{H}_{11} \mathrm{NO}$ & Others & 126.0917 & 6.94 & 2.9665 & 0.0047644 & 0.008338 & -1.14 \\
\hline $\mathrm{C} 02674$ & $\mathrm{C}_{5} \mathrm{H}_{8} \mathrm{O}_{4}$ & Others & 131.0346 & 5.05 & 2.4719 & 0.017201 & 0.026367 & -1.65 \\
\hline C09930 & $\mathrm{C}_{13} \mathrm{H}_{12} \mathrm{O}_{4}$ & Others & 233.0815 & 7.44 & 4.7745 & $1.87 \mathrm{E}-05$ & $6.03 \mathrm{E}-05$ & -1.68 \\
\hline $101228910^{*}$ & $\mathrm{C}_{18} \mathrm{H}_{24} \mathrm{O}_{8}$ & Others & 367.1387 & 8.59 & 4.6235 & $3.07 \mathrm{E}-05$ & $8.96 \mathrm{E}-05$ & -1.47 \\
\hline $10328^{*}$ & $\mathrm{C}_{8} \mathrm{H}_{9} \mathrm{~N}$ & Others & 120.0807 & 5.52 & -3.0638 & 0.003648 & 0.006604 & 1.60 \\
\hline $657272^{*}$ & $\mathrm{C}_{8} \mathrm{H}_{20} \mathrm{NO}_{6} \mathrm{P}$ & Others & 258.1104 & 0.78 & 4.7518 & $2.01 \mathrm{E}-05$ & $6.31 \mathrm{E}-05$ & -1.80 \\
\hline $44258370^{*}$ & $\mathrm{C}_{28} \mathrm{H}_{32} \mathrm{O}_{16}$ & Others & 623.1613 & 7.3 & 2.8932 & 0.0058099 & 0.009839 & -1.34 \\
\hline $\mathrm{C} 07115$ & $\mathrm{C}_{5} \mathrm{H}_{11} \mathrm{C}_{12} \mathrm{~N}$ & Others & 156.0426 & 0.81 & 2.4599 & 0.017715 & 0.026958 & -1.36 \\
\hline $11557800^{*}$ & $\mathrm{C}_{12} \mathrm{H}_{20} \mathrm{O}_{5}$ & Others & 243.1236 & 8.22 & 3.1737 & 0.0026832 & 0.005123 & -1.09 \\
\hline C07609 & $\mathrm{C}_{15} \mathrm{H}_{20} \mathrm{O}_{3}$ & Others & 249.1483 & 11.66 & 3.1625 & 0.002769 & 0.005239 & -1.25 \\
\hline $\mathrm{C} 00250$ & $\mathrm{C}_{8} \mathrm{H}_{9} \mathrm{NO}_{3}$ & Others & 168.0659 & 1.5 & 6.5337 & $4.57 \mathrm{E}-08$ & $3.43 \mathrm{E}-07$ & -1.94 \\
\hline $163490^{*}$ & $\mathrm{C}_{15} \mathrm{H}_{21} \mathrm{NO}_{7}$ & Others & 328.1395 & 5.55 & -2.6999 & 0.0096736 & 0.015996 & 2.02 \\
\hline
\end{tabular}


Supplemental Table 2. List of 89 significantly different levels of metabolites (ANOVA, $\mathrm{P} \leq 0.05$, Fisher's LSD) in the wheat flag leaves at 8 different time points $(3 \mathrm{~h}, 6 \mathrm{~h}, 9 \mathrm{~h}, 12 \mathrm{~h}, 15 \mathrm{~h}, 18 \mathrm{~h}, 21 \mathrm{~h}, 24 \mathrm{~h})$ during 24 hours of day under drought and control conditions with their P-value, false discovery rate (FDR) and significant pairs of time points.

\begin{tabular}{|c|c|c|c|}
\hline Sl\# & Metabolites & $\mathrm{p}$ value & FDR \\
\hline 1 & TRIBUFOS & $1.29 \mathrm{E}-05$ & 0.001372 \\
\hline 2 & N-ACETYL-DL-GLUTAMIC ACID & $2.03 \mathrm{E}-05$ & 0.001372 \\
\hline 3 & DIBOA-GLUCOSIDE & $2.71 \mathrm{E}-05$ & 0.001372 \\
\hline 4 & LILIOSIDE A & $8.43 \mathrm{E}-05$ & 0.003079 \\
\hline 5 & LICHENIN & 0.000101 & 0.003079 \\
\hline 6 & ALPHA-AMINOADIPATE & 0.000128 & 0.003244 \\
\hline 7 & N-HYDROXY-L-VALINE & 0.000174 & 0.003635 \\
\hline 8 & 5-GUANIDINO-3-METHYL-2-OXOPENTANOIC ACID & 0.000217 & 0.003635 \\
\hline 9 & L-CARNITINE & 0.000279 & 0.003635 \\
\hline 10 & GLUCONIC ACID & 0.000281 & 0.003635 \\
\hline 11 & EUPALITIN 3-O-SULFATE & 0.000281 & 0.003635 \\
\hline 12 & TRIBENURON METHYL & 0.000304 & 0.003635 \\
\hline 13 & STROPHANTHOBIOSE & 0.000327 & 0.003635 \\
\hline 14 & 1-INDOL-3-YLPROPANOL 3-PHOSPHATE & 0.000359 & 0.003635 \\
\hline 15 & ADENINE & 0.000434 & 0.003635 \\
\hline 16 & L-PROLINE & 0.000437 & 0.003635 \\
\hline 17 & 4Z-DECENEDIOIC ACID & 0.000465 & 0.003635 \\
\hline 18 & 2-VALERYL-SN-GLYCERO-3-PHOSPHOCHOLINE & 0.000477 & 0.003635 \\
\hline 19 & D-XYLONATE & 0.00048 & 0.003635 \\
\hline 20 & LACTOBIONIC ACID & 0.000493 & 0.003635 \\
\hline 21 & GLCN1 INO1 & 0.000502 & 0.003635 \\
\hline 22 & D-TREHALOSE & 0.000547 & 0.003776 \\
\hline 23 & 5'-O-BETA-D-GLUCOSYLPYRIDOXINE & 0.000751 & 0.004964 \\
\hline 24 & 4-HYDROXY-2-BUTYNAL & 0.000893 & 0.005583 \\
\hline 25 & 4-CARBOXYLATOMETHYLENEBUT-2-EN-4-OLIDE & 0.000918 & 0.005583 \\
\hline 26 & 3-METHYLENEOXINDOLE & 0.001308 & 0.007648 \\
\hline 27 & OXIDIZED GLUTATHIONE & 0.001402 & 0.007892 \\
\hline 28 & P-CYMENE & 0.001729 & 0.009051 \\
\hline 29 & CERHEPTARIC ACID & 0.001731 & 0.009051 \\
\hline 30 & BIS-D-FRUCTOSE 2',12,1'-DIANHYDRIDE & 0.001786 & 0.009051 \\
\hline 31 & PARTHENOLIDE & 0.001913 & 0.009379 \\
\hline 32 & D-THREO-L-GALACTO-OCTOSE & 0.003424 & 0.016057 \\
\hline
\end{tabular}




\section{Continued}

\begin{tabular}{|c|c|c|c|}
\hline 33 & GLYCERATE & 0.003633 & 0.016057 \\
\hline 34 & DIHYDRO-4,4-DIMETHYL-2,3-FURANDIONE & 0.003668 & 0.016057 \\
\hline 35 & BETA-CYMAROPYRANOSE & 0.003697 & 0.016057 \\
\hline 36 & GUANIDINOPROCLAVAMINIC ACID & 0.004071 & 0.01719 \\
\hline 37 & NICOTINAMIDE & 0.004608 & 0.018929 \\
\hline 38 & ALPHA-METHYLENE GAMMA-BUTYROLACTONE & 0.005008 & 0.020033 \\
\hline 39 & SUCROSE & 0.005287 & 0.020132 \\
\hline 40 & 1,2,3-TRIHYDROXYBENZENE & 0.005298 & 0.020132 \\
\hline 41 & L-SERINE & 0.005855 & 0.021701 \\
\hline 42 & L-PHENYLALANINE & 0.005996 & 0.021701 \\
\hline 43 & DEOXYRIBOSE & 0.006232 & 0.021781 \\
\hline 44 & CALYSTEGIN B2 & 0.006387 & 0.021781 \\
\hline 45 & ALPHA-KETOGLUTARIC ACID & 0.006491 & 0.021781 \\
\hline 46 & FRUCTOSELYSINE 6-PHOSPHATE & 0.006764 & 0.021781 \\
\hline 47 & GLUTATHIONE & 0.006947 & 0.021781 \\
\hline 48 & PRUYANASIDE B & 0.006965 & 0.021781 \\
\hline 49 & SLAFRAMINE & 0.007022 & 0.021781 \\
\hline 50 & SESBANIMIDE & 0.007351 & 0.022347 \\
\hline 51 & PALMITOLEIC ACID & 0.008661 & 0.025812 \\
\hline 52 & GLUCOGALLIN & 0.009096 & 0.026576 \\
\hline 53 & ADENOSINE & 0.009305 & 0.026576 \\
\hline 54 & GUANINE & 0.009442 & 0.026576 \\
\hline 55 & D-ARABINOSE & 0.010386 & 0.028704 \\
\hline 56 & D-GLUCONO-1,5-LACTONE & 0.011433 & 0.031033 \\
\hline 57 & 2ALPHA-D-MANNOSYL-D-GLYCERATE & 0.012334 & 0.032891 \\
\hline 58 & 5-OXO-L-PROLINE & 0.012912 & 0.033838 \\
\hline 59 & PYRIDOXAL & 0.015342 & 0.039525 \\
\hline 60 & D-GLUCOSE & 0.015823 & 0.040084 \\
\hline 61 & GONIOTHALENOL & 0.016758 & 0.041757 \\
\hline 62 & 5-SULFOSALICYLATE & 0.018782 & 0.046046 \\
\hline 63 & 12-HYDROXYJASMONIC ACID & 0.019325 & 0.046626 \\
\hline 64 & SEBACIC ACID & 0.022335 & 0.053045 \\
\hline 65 & CARBETAMIDE & 0.023491 & 0.054932 \\
\hline 66 & L-GLUTAMINE & 0.024783 & 0.056838 \\
\hline 67 & DIMETHYLMALEATE & 0.025162 & 0.056838 \\
\hline
\end{tabular}




\section{Continued}

\begin{tabular}{|c|c|c|c|}
\hline 68 & CLITHIONEINE & 0.025427 & 0.056838 \\
\hline 69 & NICOTINATE & 0.026448 & 0.0578 \\
\hline 70 & ALANCINE & 0.026989 & 0.0578 \\
\hline 71 & CITRININ & 0.027199 & 0.0578 \\
\hline 72 & L-ALPHA-GLYCEROPHOSPHOCHOLINE & 0.027379 & 0.0578 \\
\hline 73 & 4-AMINOBUTANOATE & 0.028627 & 0.059608 \\
\hline 74 & N-ACETYL-D-FUCOSAMINE & 0.03025 & 0.06146 \\
\hline 75 & L-TRYPTOPHAN & 0.030326 & 0.06146 \\
\hline 76 & INDOLINE & 0.031295 & 0.062591 \\
\hline 77 & DECYL BETA-D-MALTOPYRANOSIDE & 0.0318 & 0.062773 \\
\hline 78 & D--RAFFINOSE & 0.03439 & 0.066434 \\
\hline 79 & 2-HYDROXYPHENYLACETIC ACID & 0.034528 & 0.066434 \\
\hline 80 & D-SACCHARIC ACID & 0.035523 & 0.067494 \\
\hline 81 & AURANTIO-OBTUSIN BETA-D-GLUCOSIDE & 0.038175 & 0.071636 \\
\hline 82 & PIPECOLATE & 0.039397 & 0.073028 \\
\hline 83 & LINOLEATE & 0.040494 & 0.074158 \\
\hline 84 & URACIL & 0.044783 & 0.080305 \\
\hline 85 & SPERMIDINE & 0.045363 & 0.080305 \\
\hline 86 & LINAMARIN & 0.045435 & 0.080305 \\
\hline 87 & QUINATE & 0.046014 & 0.080393 \\
\hline 88 & PANDANGOLIDE & 0.047594 & 0.081527 \\
\hline 89 & SEDOHEPTULOSE & 0.047736 & 0.081527 \\
\hline
\end{tabular}

